\title{
Gut microbiota modulates lung fibrosis severity following acute lung injury
}

\section{Ozioma Chioma}

Vanderbilt University Medical Center

\section{Laura Hesse}

Vanderbilt University Medical Center

\section{Elizabeth Mallott}

Northwestern University https://orcid.org/0000-0001-5446-8563

\section{Austin Chapman}

Vanderbilt University Medical Center

Joseph Van Amburg

Vanderbilt University Medical Center

Hongmei Wu

Vanderbilt University Medical Center

Binal Shah-Gandhi

Vanderbilt University Medical Center

\section{Blanca Piazuelo}

Vanderbilt University Medical Center https://orcid.org/0000-0002-0000-1324

Joyce Johnson

Vanderbilt University School of Medicine

Gordon Bernard

Vanderbilt University Medical Center,

\section{Sobha Bodduluri}

University of Louisville

\section{Steven Davison}

University of Louisville

\section{Bodduluri Haribabu}

University of Louisville School of Medicine

\section{Seth Bordenstein}

Vanderbilt University https://orcid.org/0000-0001-7346-0954

Wonder Drake ( $\boldsymbol{\nabla}$ wonder.drake@vumc.org )

Vanderbilt University Medical Center 
Keywords: lung fibrosis, acute lung injury, gut microbiota

Posted Date: August 4th, 2021

DOI: https://doi.org/10.21203/rs.3.rs-622623/v1

License: (c) (1) This work is licensed under a Creative Commons Attribution 4.0 International License. Read Full License 


\section{Gut microbiota modulates lung fibrosis severity following acute lung injury}

Ozioma S. Chioma ${ }^{\mathrm{a} 1}$, Laura E. Hesse ${ }^{\mathrm{a} 2}$, Elizabeth K. Mallott ${ }^{3,4}$, Austin Chapman ${ }^{1}$, Joseph C. Van Amburg ${ }^{1}$, Hongmei Wu${ }^{1}$, Binal Shah-Gandhi ${ }^{1}$, M. Blanca Piazuelo 5 ; Joyce Johnson ${ }^{2}$, Gordon R. Bernard $^{6}$, Sobha R. Bodduluri ${ }^{7}$, Steven Davison ${ }^{8}$, Bodduluri Haribabu ${ }^{7}$, Seth R. Bordenstein ${ }^{2,3,4}$, Wonder P. Drake*1, 2

Affiliations:

${ }^{1}$ Division of Infectious Disease, Department of Medicine, Vanderbilt University School of Medicine, Nashville, TN

${ }^{2}$ Department of Pathology, Microbiology, and Immunology, Vanderbilt University School of Medicine, Nashville, TN

${ }^{3}$ Department of Biological Sciences, Vanderbilt University, Nashville, TN

${ }^{4}$ Vanderbilt Microbiome Initiative, Vanderbilt University, Nashville, TN

${ }^{5}$ Division of Gastroentrology, Department of Medicine, Vanderbilt University School of Medicine, Nashville, TN

${ }^{6}$ Division of Pulmonary and Critical Care, Department of Medicine, Vanderbilt University School of Medicine, Nashville, TN

${ }^{7}$ Department of Microbiology and Immunology, University of Louisville School of Medicine, Louisville, KY

${ }^{8}$ Comparative Medicine Research Unit, University of Louisville School of Medicine, Louisville, KY

aauthors contributed equally

*corresponding author: wonder.drake@vumc.org 


\begin{abstract}
Independent reports note the significance of gut microbiota on lung disease severity; however, studies using murine models to define the role of the gut microbiome in pulmonary fibrosis progression are missing. We used the bleomycin murine model to quantify lung fibrosis in C57BL/6J mice housed in germ-free, animal biosafety level 1 (ABSL-1), or animal biosafety level 2 (ABSL-2) environments. Mice housed in gnotobiotic facilities are protected from bleomycininduced pulmonary fibrosis, while ABSL-1 and ABSL-2 mice develop mild fibrosis and severe lung fibrosis, respectively. Metagenomic analysis of the gut microbiota revealed greater microbial diversity in ABSL-1 compared to ABSL-2 mice, with an increased presence of Lactobacilli and Bifidobacterium in ABSL-1 mice. Flow cytometric analysis of single-cell lung suspensions revealed enhanced IL-6/STAT3 /IL-17A signaling in CD4+ T cells of ABSL-2 mice, compared to ABSL-1 or germ-free mice. Fecal microbiota transplantation (FMT) of low microbial diverse stool (ABSL-2) into germ-free mice before bleomycin administration recapitulated the severe fibrosis phenotype, whereas FMT of ABSL-1 stool induced minimal fibrosis. These findings strongly support a causal role of the gut microbiota in augmenting pulmonary fibrosis severity after acute lung injury.
\end{abstract}

Word count: 184 


\section{Introduction}

Accumulating evidence suggests that the gut microbiota have a profound impact on lung pathophysiology, such as the impact of early gut microbiome on childhood asthma ${ }^{1}$. Fibrotic lung disease, a major cause of morbidity and mortality worldwide, occurs when fibroblasts are stimulated to overproduce collagen and other extracellular matrix components in response to inflammatory signals ${ }^{2}$. Current treatments rely on slowing disease progression, yet the mortality rate for some fibrotic lung diseases remains unacceptably high ${ }^{3-5}$. Studies in patient cohorts with idiopathic pulmonary fibrosis (IPF) have described altered lung microbiota, and correlate high lung bacterial burden with enhanced disease ${ }^{6,7}$. Additionally, metagenomic sequencing of fecal samples from autoimmune fibrotic lung diseases, such as systemic sclerosis and silicosis, revealed dysbiosis and lack of gut community diversity ${ }^{8,9}$. Despite these striking clinical correlations, mechanistic studies using murine models to define the role of the gut microbiota in pulmonary fibrosis development and progression are missing.

A growing body of literature supports a gut-lung axis, where processes occurring in the gut associate with outcomes of lung pathologies ${ }^{10,11}$. The gut microbiota can influence the lung inflammatory environment in several ways. Production of inflammatory cytokines such as IL-6, which play a role in fibrosis development, can be induced by gut microbes ${ }^{12-14}$. Enhanced IL-6 signaling has been shown to contribute to interstitial lung disease (ILD) pathogenesis, through the IL-6/STAT3/IL-17A signaling pathway ${ }^{15-17}$. Conversely, microbial metabolites, such as the short chain fatty acid (SCFA) butyrate, produced by Lactobacilli and Bifidobacterium, can have antiinflammatory properties and bias $\mathrm{T}$ cells toward a Th1 or $\mathrm{T}$ regulatory rather than Th17 phenotype $^{18,19}$. These contrasting roles for the gut microbiota in immune activation suggest that 
the specific microbial composition of gut communities is important for enhancing or suppressing pathogenic immune responses contributing to lung fibrosis.

This study used direct modulation of the gut microbiota via rearing environments, fecal microbiota transplantation (FMT) in a bleomycin-induced murine model to demonstrate that a functional gutlung axis modulates lung fibrosis. While germ-free (GF) mice developed significantly less lung fibrosis than ABSL-1 or ABSL-2 housed mice, FMT of the ABSL-2 stool into germ-free mice before bleomycin administration recapitulated the severe fibrosis phenotype. These results specify that degrees of lung fibrosis severity can be modulated by the absence and/or diversity of the gut microbiota.

\section{Results}

Rearing environment impacts severity of pulmonary fibrosis

To assess the contribution of the gut microbiota to lung fibrosis severity, we housed mice in three distinct environments: GF (no microorganisms), ABSL-1 (no experiments involving infectious agents; mice possess only commensal organisms), and ABSL-2 (experiments involving infectious agents of moderate potential hazard to personnel are present in environment). None of the mice in our investigations were ever infected; instead, 6-week-old male and female mice in each environment were administered either saline or bleomycin intranasally and monitored for 14 days. When given intranasal bleomycin, all GF mice exhibited 100\% survival (Fig. 1a). ABSL-2 mice administered bleomycin exhibited the greatest mortality, and ABSL-1 survival was intermediate of GF and ABSL-2 mice (Fig. 1a). Significant distinctions in survival were noted among the ABSL- 2 and GF mice $(p=0.026$, log-rank MantelCox test). When treated with saline intranasally, mice in all housing cohorts had similar survival 
plots (Fig. S1a), did not experience significantly different weight loss (Fig. S1b) and had similar lung collagen content (Fig. S1c), suggesting no acute lung injury. There were significant morbidity distinctions, in terms of weight loss, to mice administered bleomycin. Except for mice housed in gnotobiotic facilities, ABSL-1 and ABSL-2 mice administered saline demonstrated significantly less weight loss than those receiving bleomycin $(\mathrm{p}<0.0001$ and $\mathrm{p}<0.01$, respectively; one-way ANOVA with Tukey's multiple comparison's test) (Fig 1b). Also, GF mice receiving bleomycin exhibited significantly less weight loss compared to ABSL-1 mice ( $\mathrm{p}<0.001$, one-way ANOVA with Tukey's multiple comparison's test), as well as ABSL-2 mice ( $<<0.01$, one-way ANOVA with Tukey's multiple comparison's test) (Fig. 1b). Qualitative and quantitative histologic assessments of the lung following bleomycin administration in the three different rearing environments were conducted. To analyze fibrosis parameters, we conducted pulmonary trichrome staining and measured the collagen content by the Sircol assay. Representative hematoxylin and eosin (H\&E) and trichrome sections of bleomycin-treated mice under the three rearing conditions (Fig. 1c). Fibrosis severity was less severe by Ashcroft scoring in GF mice compared to ABSL-1 mice, as well as compared to ABSL-2 mice (Fig. 1d). Notably, quantification of collagen content revealed that ABSL-2 mice had significantly higher lung collagen content than GF ( $\mathrm{p}<0.0001$, one-way ANOVA with Tukey's multiple comparison's test) or ABSL-1 mice ( $\mathrm{p}<0.0001$, one-way ANOVA with Tukey's multiple comparison's test). The ABSL-1 mice had intermediate lung fibrosis of GF and ABSL-2 mice ( $p<0.01$, one-way ANOVA with Tukey's multiple comparison's test) (Fig. 1e). Remarkably, the GF mice administered bleomycin had pulmonary collagen contents similar to mice administered intranasal saline (Figure 1e). These findings indicate that the environment in which the murine host lives contributes to lung fibrosis severity. 
Lack of gut microbial diversity associates with severity of lung fibrosis

To investigate the hypothesis that the gut microbiota is an important contributor to the differences in fibrosis severity between ABSL-1 and ABSL-2 housed mice, we performed metagenomic analysis on fecal pellets from mice in each housing cohort. We did not detect microorganisms in the stool of GF mice by sequencing and culture as expected. Shannon alpha diversity, a measure of species richness and evenness, was significantly higher in ABSL-1 mice compared with ABSL2 mice using a Wilcoxon rank sum test $(\mathrm{W}=166, \mathrm{p}=0.010)$ (Fig. 2a). Shannon alpha diversity did not differ between treatments (bleomycin vs. saline) using a Wilcoxon rank sum test (W=367, $\mathrm{p}=0.278$ ), suggesting that bleomycin treatment does not alter the diversity of the gut microbiota. We compared the relative abundance of phyla using a generalized linear model (GLM) with a negative binomial distribution. ABSL-1 mice had significantly higher relative abundances of Proteobacteria $\left(\mathrm{GLM}, \chi^{2}=20.221, \mathrm{p}<0.001\right)$ and Verrucomicrobia $\left(\mathrm{GLM}, \chi^{2}=4.487, \mathrm{p}=0.034\right)$ (Fig. 2b). Increased proteobacteria abundance has been linked with both inflammatory gut and airway environments, as in Crohn's disease ${ }^{20}$, asthma ${ }^{21}$ and after lung transplantation ${ }^{22}$. Beta diversity differences between ABSL-1 and ABSL-2 microbiota compositions also differed significantly when analysis was conducted using both the Bray-Curtis dissimilarity metric (Fig. 2c) and Jaccard index (Fig. S2) that account for taxon abundance variation and presence/absence of taxa, respectively (PERMANOVA, Bray-Curtis: $F_{2,47}=4.676, \mathrm{R}^{2}=0.089, \mathrm{p}=0.006$; Jaccard: $\mathrm{F}_{2,47}=4.735$, $\mathrm{R}^{2}=0.090, \mathrm{p}=0.003$ ). Treatment (bleomycin vs. saline) was not significantly associated with taxonomic composition using either metric, indicating that bleomycin treatment does not alter the gut microbiota taxonomic composition (Bray-Curtis: $F_{2,47}=0.696, \mathrm{R}^{2}=0.013$, $\mathrm{p}=0.546$; Jaccard: $\mathrm{F}_{2,47}=0.992, \mathrm{R}^{2}=0.019, \mathrm{p}=0.390$ ) (Fig. $2 \mathrm{c}$ ). 
Using linear discriminant analysis (LDA) to examine species-level differences in the gut microbiota, 10 taxa were overrepresented in ABSL-1 mice and 5 taxa were overrepresented in ABSL-2 mice (Fig. 2d). The overrepresented taxa in ABSL-2 mice, included Lachnospiraceae bacterium A2, Lachnospiraceae bacterium 28-4, Firmicutes bacterium ASF500, and Romboutsia ilealis. Relative abundance of Firmicutes in the lung microbiota of bleomycin-treated mice with fibrosis has been reported ${ }^{7}$. Species overrepresented in ABSL-1 mice include Staphylococcus nepalensis, Dubosiella newyorkensis, Acetatifactor muris, Lactobacillus animalis, Lactobacillus murinus, and Acutalibacter muris. Of note, Lactobacillus species, which are overrepresented in ABSL-1 mice, have been shown to reduce IL-17A-mediated disease severity in other experimental animal models ${ }^{23-25}$, and is associated with significant reductions in respiratory symptoms ${ }^{26}$.

Beta diversity differences in metagenomic functional gene profiles between rearing environment and treatment conditions were also examined using Jaccard distances (Fig. 3a and 3b) and BrayCurtis dissimilarities (Fig. S3a and S3b). Functional profiles across gene family and MetaCyc reaction pathway abundances were significantly associated with environment and treatment specifically when examining Jaccard distances (Fig. 3c). MetaCyc reaction pathway abundances differed significantly between the gut microbiota composition of ABSL-1 and ABSL-2 mice (Jaccard: $F_{2,47}=3.055, R^{2}=0.058, p<0.001$ ) (Fig. 3c). Similarly, rearing environment was significantly associated with gene family profiles (PERMANOVA; Jaccard: $F_{2,47}=7.030$, $\mathrm{R}^{2}=0.125, \mathrm{p}<0.001$ ) (Fig. 3b). A difference between rearing environments only when using Jaccard distances, and not Bray-Curtis dissimilarities, indicates that low abundance pathways are driving the differences between rearing environments. Shannon diversity was higher in ABSL-1 mice for both MetaCyc reaction pathways $(\mathrm{W}=544, \mathrm{p}<0.001)$ and gene families $(\mathrm{W}=149, \mathrm{p}=0.003)$, but did not differ between saline and bleomycin treatments. GLMs did not identify any differentially 
abundant pathways between ABSL-1 or ABSL-2 mice. However, LDA identified 24 pathways that were overrepresented in ABSL-1 mice and one pathway that was overrepresented in ABSL-2 mice (Fig. 3d). Microbial community compositional and functional differences between mice in separate rearing groups with differing amount of fibrosis support the hypothesis that the gut microbiota may directly contribute to the degree of lung fibrosis.

\section{Gut microbial diversity and taxa associate with proinflammatory lung responses}

To determine the immunologic drivers of lung fibrosis under distinct rearing environments, we performed flow cytometric analysis of single cell lung suspensions obtained from GF, ABSL-1, and ABSL-2 mice 14 days after inoculation with either saline or bleomycin. We noted no difference in CD4+IL-6+ T cells in the saline-treated control group between the three facilities (Fig. S4a); however, with bleomycin treatment, there was a significant increase in CD4+IL-6+ T cells in ABSL-2 mice compared to GF or ABSL-1 mice (Fig. 4a; representative histogram in Fig. S5a). The same trend occurred for the IL-6 co-receptor, gp130, where bleomycin-treated mice in the ABSL-2 cohort had the highest percentage of CD4+ gp130+ T cells (Fig. $4 \mathrm{~b}$ representative histograms in Fig. S5b). We previously reported that PD-1+ Th17 cells contribute to the development of lung fibrosis. We also noted nonsignificant levels of Programmed Death-1+(PD1) Th17 cells in GF and ABSL-1 mice, compared to ABSL-2 mice (Figure 4c). Representative histograms and gating strategy are shown (Fig. 4d, e, S5c). Bleomycin treatment increases pSTAT3 expression in CD4+ T cells in the ABSL-2 cohort mice (Fig. 4f). There was no difference across any treatment or housing cohort in the expression of IL-23R (Fig. S4b), suggesting that increased pSTAT3 is due to enhanced IL-6 rather than IL-23 signaling. The distinctions in the percentages of CD4+IL-17A $+\mathrm{T}$ cells in GF and ABSL-2 mice support microbiota-induced alterations to the IL-6/ STAT3/IL-17A pathway in pulmonary fibrosis. 
As there were differences between immune variables in the mice with different microbiota, we looked for associations between individual taxa abundances and immunological variables using Spearman correlation tests. Several taxa, including members of the Firmicutes and Proteobacteria, were found to be negatively correlated with multiple immunological values (Table 1), and Romboutsia ilealis was positively correlated with all immunological values (Table 1). The species negatively correlated with immune activation include Lactobacillus species, which have been shown to produce butyrate that dampens Th17 polarization $^{18,19}$.

\section{Reconstitution of low diversity gut microbiota enhances pulmonary fibrosis}

To test the hypothesis that the gut microbiota causes fibrosis severity, we performed fecal microbiota transplantation (FMT) experiments. GF mice were gavaged every other day with ABSL-1 or ABSL-2 stool for one week prior to bleomycin inoculation and during the 14 days following the induction of lung injury. We observed a trend in increased weight loss at day 14 compared to untreated GF mice in mice treated with ABSL-2 stool, but not ABSL-1 stool (Fig. 5a). This corresponded with a significant increase in lung collagen content in the GF mice that underwent FMT of ABSL-2 stool. No increase in lung fibrosis was detected among GF mice following FMT of ABSL-1 stool (Fig. 5b). This data suggests that lung injury alone is not sufficient to induce fibrosis but that bacteria capable of inducing profibrotic cytokine expression also need to be present in the gut.

\section{Discussion}

Using the murine model of pulmonary fibrosis under distinct rearing environments, we establish the impact of variation in gut microbial diversity on lung fibrosis severity. In particular, low gut 
microbial diversity was present among ABSL-2 mice that possessed severe lung fibrosis, whereas higher microbial diversity was present among ABSL-1 mice with less lung fibrosis following bleomycin administration (Fig. 2). Despite intranasal bleomycin treatment, GF mice did not develop lung fibrosis. FMT of ABSL-2 stool into GF mice before bleomycin treatment enhanced fibrosis severity, directly linking gut microbial composition with disease outcomes. (Fig. 5).

We observed protection from bleomycin-induced acute lung injury in GF mice (Fig. 1), supporting the hypothesis that acute lung injury alone is not sufficient to induce lung pathology, but rather "two-hits" are necessary to induce pulmonary fibrosis: 1) acute lung injury (ALI) and 2) presence of microorganisms capable of inducing profibrotic cytokine expression, such as IL-17A. ABSL2 mice do not spontaneously develop lung fibrosis, even if housed for seven months (data not shown). Remarkably, GF mice demonstrate minimal collagen deposition following intranasal bleomycin instillation. The significance of the gut microbiota to lung fibrosis progression was further supported by FMT of ABSL-2 stool into GF mice to recapitulate fibrotic lung disease severity. FMT of ABSL-1 stool into GF mice did not induce significant lung fibrosis. A recent clinical trial noted reductions in the incidence of upper respiratory tract infections among patients randomized to probiotics containing Lactobacilli and Bifidobacteria compared to placebo ${ }^{26}$, further supporting the importance of the gut-lung axis in lung disease.

Investigation of the pulmonary inflammatory milieu among the different cohorts of mice provides an interesting linkage between profibrotic cytokine expression and reduced microbial diversity. Independent investigators support the capacity of the gut microbiota to induce systemic inflammation, specifically increased IL-6, IFN- $\gamma$ and IL-17A, through mechanisms such as 1) formation of IsoLG-protein adducts in dendritic cells, which drive IFN- $\gamma$ and IL-17A production by $\mathrm{T}$ cells ${ }^{27}$; 2) augmentation of gut permeability allows translocation of bacterial endotoxin 
(lipopolysaccharide, LPS) from the gut microbiota to blood circulation, leading to microbiotadependent interleukin-17A (IL-17A) secretion from lamina propria derived Th17 cells ${ }^{28}$; or 3) Reduced expression of short chain fatty acids, such as butyrate, by the gut microbiota enhances Th17 cell expansion ${ }^{29}$. It is also noteworthy that microbial strains shown to improve gut permeability, such as Bifidobacterium, were most prevalent in the ABSL-1 stool (Fig. 2). Importantly, many of the organisms enriched in the ABSL-1 cohort, including Dubosiella newyorkensis, Staphylococcus nepalensis, and two Lactobacillus species were also correlated with decreased immune activation (Table 1). The only species that had a positive correlation with immune activation, Romboutsia ilealis, was enriched in the ABSL-2 mice. Romboutsia species are associated with the metabolic changes associated with obesity ${ }^{30}$, which serves as an independent risk factor for pulmonary progression in the ILD sarcoidosis ${ }^{31}$. Further investigation of the impact of specific microbial species such as Romboutsia on metabolic disorders associated with lung fibrosis is warranted. Metagenomic analysis also revealed relatively higher abundance of Lactobacillus species in ABSL-1 housed mice (Fig. 2), which demonstrated fewer Th17 cells (Fig. 4). Various Lactobacilli species can reduce IL-17A expression via TLR2/4 in epithelial cells $^{25}$ or through production of metabolites such as butyrate ${ }^{23}$. SCFA, like butyrate, reduce inflammatory conditions following insult through their capacity to decrease IL-17A synthesis, as well as promote IL-10 generation through T regulatory cell development ${ }^{19}$. Modulation of the gut microbiota of mice by oral gavage of Lactobacillus reuteri protected mice from multiple sclerosislike disease symptoms by decreasing IL-17A levels ${ }^{24}$. Future investigation of the impact of microbial diversity on lung fibrosis is warranted, including assessing the ability of probiotic treatment with Lactobacillus spp. to reduce disease severity in patients with lung fibrosis. 
In addition to identifying members of the gut microbiota that may contribute to or protect from severe lung fibrosis, our investigation suggests that increased diversity in gut community composition is beneficial with respect to pulmonary disease severity (Figs. 1, 5). This observation follows with the ecological principle that more diverse ecosystems are more resistant to perturbations than less diverse systems. Diversity has been shown to be beneficial for both community stability and functionality over time, whether members of the ecosystem are animals, plants, or microorganisms ${ }^{32-35}$. The human gut microbiota represents a thriving ecosystem with taxonomic and functional diversity within and between individuals ${ }^{36}$. Fecal microbiota transplantation (FMT) has been explored clinically to increase gut microbial diversity to combat complications such as those occurring after allogeneic hematopoietic stem cell transplantation ${ }^{37}$ and Clostridium difficile infection ${ }^{38}$. Additional murine studies using FMT have demonstrated increased murine survival after acute liver injury and during acute respiratory distress syndrome by decreasing IL-17A levels ${ }^{39,40}$. Our investigations add to this growing body of FMT literature, suggesting that functional and taxonomic diversity in the gut microbiota of patients with lung fibrosis may improve their clinical outcomes, and that decreased gut microbial diversity may predispose individuals to more severe manifestations of fibrotic lung disease (Fig 6). Molecular examination of ABSL-1 stool demonstrated that there were some proinflammatory bacteria present, such as proteobacteria which has been linked with both inflammatory gut and airway environments asthma ${ }^{21}$ and after lung transplantation ${ }^{22}$; however, companion microorganisms, such as lactobacilli, limit their capacity to induce lung disease severity.

There are limitations to this investigation. While our sequencing studies focused on the bacterial members of the gut community, the potential role of fungal and viral contributors to the gut microbiota's impact on pulmonary fibrosis cannot be discounted. In addition, other components 
of stool, including host-derived cells and metabolites, are transferred to the naïve host during $\mathrm{FMT}^{41}$. Also, this study did not probe lung microbial communities among mice in the different rearing environments; altered lung microbiota have been noted to be important in ILD patients ${ }^{7,42}$. Further investigation of the influence of the gut microbiota on the lung microbial community and vice versa is warranted.

Overall, our study implicates the low-diversity gut microbiota as a contributor of pulmonary fibrosis severity through their capacity to activate the IL-6/STAT3/IL-17A pathway. These findings support that acute lung injury in a host with low microbial diversity leads to more severe lung disease, compared to hosts with increased microbial diversity. Validation of the potential benefit of enhancing gut microbial diversity in ILD patients is warranted.

\section{Methods}

\section{Murine model of lung fibrosis}

We reared mice in either germ-free (gnotobiotic conditions with no microbial interactions, animal biosafety level 1 (ABSL-1) experimental conditions in which no infections were conducted, or animal biosafety level 2 (ABSL-2) environments in which infections with pathogenic microorganisms occur. None of the mice in any of the facilities underwent inoculation with any microorganisms; only bleomycin was administered intranasally to induce lung fibrosis. For the murine model of bleomycin-induced pulmonary fibrosis, 6- to 8-week-old C57BL/6J mice from Jackson Labs weighing approximately 17-22g were used. Mice were anesthetized by intraperitoneal injection of $80 \mu \mathrm{l}$ of $20 \mathrm{mg} / \mathrm{ml} \mathrm{Ketamine} / 1.8 \mathrm{mg} / \mathrm{ml}$ Xylazine solution, then $75 \mu \mathrm{l}$ containing 0.04 Units of bleomycin (Novaplus Lake Forest IL) in saline ( $0.9 \%$ sodium chloride) (Hospira Inc, Lake forest IL) as control were administrated intranasally to mice in each housing 
cohorts as previously described ${ }^{43}$. Lungs were harvested for histology, flow cytometry, or singlecell isolation as previously described ${ }^{44}$. All murine procedures were performed according to the protocol approved by the Institutional Animal Care and Use Committee at Vanderbilt University Medical Center (protocol \#M1700043; ABSL-1 and ABSL-2 facilities, and FMT experiments) or University of Louisville (protocol \#20786; Germ-free facilities). Germ-free isolators at the University of Louisville are sampled (swabs of the environment plus fresh fecal pellets from at least 3-4 random cages) and cultured aerobically on Chocolate Agar and TSA w/ 5\% Sheep Blood Agar at $37^{\circ} \mathrm{C}$ and anaerobically on Anaerobic PEA Agar, LKV/BBE Agar (Laked Kanamycin Vancomycin/Bacteroides Bile Esculin) and Brucella Agar at $37^{\circ} \mathrm{C}$ each time the isolator is opened (roughly once/week but opened as few times as possible). No microbial growth was detected in any isolator used in this study.

\section{Metagenomic analysis of gut microbiota}

Fecal pellets were collected from mice in each housing cohort and genomic DNA (gDNA) was extracted with the Qiagen DNAeasy extraction kit (Qiagen, Valencia, CA) according to the manufacturer's instructions. The gDNA concentration and quality were confirmed using the Bioanalyzer 2100 system (Agilent, Santa Clara, CA).

Paired end sequences were trimmed, quality-filtered, and host contaminants were removed in KneadData (v.0.7.6) using default values and the C57BL mouse reference database. Taxonomic composition was profiled using MetaPhlAn (v.3.0.4) (5) $^{45}$ and functional composition was profiled using HUMAnN3 (v3.0.0) ${ }^{46}$. Samples with less than 10 million sequence reads remaining after quality filtering and removing host reads were removed prior to downstream analysis. Permutational multivariate analysis of variance (PERMANOVA) was used to assess the effects of 
both housing (ABSL-1 vs. ABSL-2) and treatment (saline vs. bleomycin) on Bray-Curtis dissimilarity and Jaccard distance matrices constructed from species-level relative abundance tables. PERMANOVAs were performed using the vegan package in $\mathrm{R}^{47,48}$. Wilcoxon Rank Sum tests in R were used to examine differences in Shannon diversity between the ABSL-1 and ABSL2 environments. We used linear discriminant analysis in $\mathrm{LEfSe}^{49}$ to identify differentially abundant taxa and pathways across both housing and treatment groups. Generalized linear models (GLMs) using a negative binomial distribution using the $\operatorname{glmm} T M B$ package in $\mathrm{R}^{50}$ were additionally used to identify differentially abundant taxa and functional pathways. Spearman correlations were used to examine associations between individual taxa abundances and physiological variables. False discovery rate (FDR) corrected p-values were calculated using the fdrtool package in $\mathrm{R}^{51}$ and are reported for all GLMs and Spearman correlations. Code for all analyses can be found at github.com/emallott/PulmonaryFibrosisMicrobiota.

Flow cytometry

All flow cytometry experiments were acquired with a LSR-II flow cytometer (BD Biosciences), and information on all antibodies used in this study is listed in Supplemental Table 1. Live cells were gated based on forward and side scatter properties, and surface staining of cells was performed as previously described ${ }^{52}$. Th17 cells were identified using flow cytometry as previously described ${ }^{53}$. Cells were gated on singlets, live CD3+ and CD4+ cells. Data analysis was performed using FlowJo software (Tree Star, Ashland, OR). A minimum of 50,000 events were acquired per sample.

Fecal Microbiota Transplantation Experiments 
Fecal pellets were collected from mice housed in the ABSL-1 and ABSL-2 facilities. These pellets were suspended in sterile PBS to create fecal slurries at 100mg feces/mL PBS. Germ-free mice were given $100 \mathrm{uL}$ of the fecal slurry by oral gavage every other day, beginning one week before intranasal inoculation with bleomycin. The germ-free mice gavaged with stool were also fed nonsterile food that originated from either the ABSL-1 or ABSL-2 facility, to match the stool received. Mice were humanely euthanized, and tissues collected 14 days after bleomycin treatment.

\section{Statistics}

Multiple-group comparisons were performed using a one-way analysis of variance (ANOVA) with Tukey's post hoc test. Statistical analysis for all figures was carried out using Prism version 7.02 (GraphPad Software). For a result to be considered statistically significant, a $P$ value of less than 0.05 was used.

\section{Acknowledgements}

This work was supported by grants NIH K24 HL127301-01, R56 RHL149129-01A1, and an Ellen Dreiling Research Fund Endowment to W.P.D and the Vanderbilt Microbiome Initiative. O.S.C is supported by T32 Grant T32AR059039-10. The germ-free mice are bred and housed at the at University of Louisville Functional Microbiomics Core facility supported by NIH/NIGMS CoBRE grant (P20GM125504). S.R.B, S.D and B.H are supported by P20GM1125504, as well as at Vanderbilt University School of Medicine Animal Facility. The model was created using BioRender.

\section{Author Contributions}


O.S.C. and L.E.H. designed and performed experiments, analyzed data, and wrote the manuscript. E.K.M. performed the metagenomic analysis. A.C., J.C.V., H.W., and B.S. performed experiments. S.R. Bordenstein and G.R.B. contributed to the intellectual content and editing of the manuscript and study design. S.D., S.R. Bodduluri, and H.B. aided with the germ-free experiments. W.P.D. conceived the study, designed experiments, analyzed data, and wrote the manuscript.

Table 1. Spearman correlation associations between individual taxa abundances and immunological variables. Spearman correlation coefficients and FDR-corrected p-values are reported. NR = no significant relationship after FDR correction.

\begin{tabular}{|c|c|c|c|}
\hline & $\begin{array}{c}\text { CD4+ PD-1+ IL-17A+ } \\
\text { T cells }\end{array}$ & $\begin{array}{c}\text { CD4+ pSTAT3+ T } \\
\text { cells }\end{array}$ & $\begin{array}{c}\text { CD4+ gp130+ } \\
\text { cells }\end{array}$ \\
\hline $\begin{array}{l}\text { Dubosiella newyorkensis } \\
\text { (Firmicutes) }\end{array}$ & $\begin{array}{l}\rho=-0.548 \\
p_{\text {fdr }}=0.011\end{array}$ & NR & NR \\
\hline $\begin{array}{l}\text { Lactobacillus animalis } \\
\text { (Firmicutes) }\end{array}$ & NR & $\begin{array}{l}\rho=-0.619 \\
p_{f d r}=0.012\end{array}$ & $\begin{array}{l}\rho=-0.532 \\
p_{\text {fdr }}=0.029\end{array}$ \\
\hline $\begin{array}{l}\text { Lactobacillus murinus } \\
\text { (Firmicutes) }\end{array}$ & NR & $\begin{array}{l}\rho=-0.619 \\
p_{\text {fdr }}=0.001\end{array}$ & $\begin{array}{l}\rho=-0.541 \\
p_{\mathrm{fdr}}=0.029\end{array}$ \\
\hline $\begin{array}{l}\text { Parasutterella } \\
\text { excrementihominis } \\
\text { (Proteobacteria) }\end{array}$ & NR & NR & $\begin{array}{l}\rho=-0.585 \\
p_{\text {fdr }}=0.029\end{array}$ \\
\hline $\begin{array}{l}\text { Proteobacteria bacterium } \\
\text { CAG.139 (Proteobacteria) }\end{array}$ & NR & NR & $\begin{array}{l}\rho=-0.564 \\
p_{\text {fdr }}=0.029\end{array}$ \\
\hline Romboutsia ilealis (Firmicutes) & $\begin{array}{l}\rho=0.412 \\
p_{\text {fdr }}=0.043\end{array}$ & $\begin{array}{l}\rho=0.599 \\
p_{\text {fdr }}=0.012\end{array}$ & $\begin{array}{l}\rho=0.542 \\
p_{\text {fdr }}=0.029\end{array}$ \\
\hline $\begin{array}{l}\text { Staphylococcus nepalensis } \\
\text { (Firmicutes) }\end{array}$ & $\begin{array}{l}\rho=-0.407 \\
p_{\text {fdr }}=0.044\end{array}$ & $\begin{array}{l}\rho=-0.608 \\
p_{\text {fdr }}=0.012\end{array}$ & $\begin{array}{l}\rho=-0.528 \\
p_{\text {fdr }}=0.029\end{array}$ \\
\hline $\begin{array}{l}\text { Turicimonas muris } \\
\text { (Proteobacteria) }\end{array}$ & NR & NR & $\begin{array}{l}\rho=-0.511 \\
p_{\text {fdr }}=0.033\end{array}$ \\
\hline
\end{tabular}


Figure 1

a

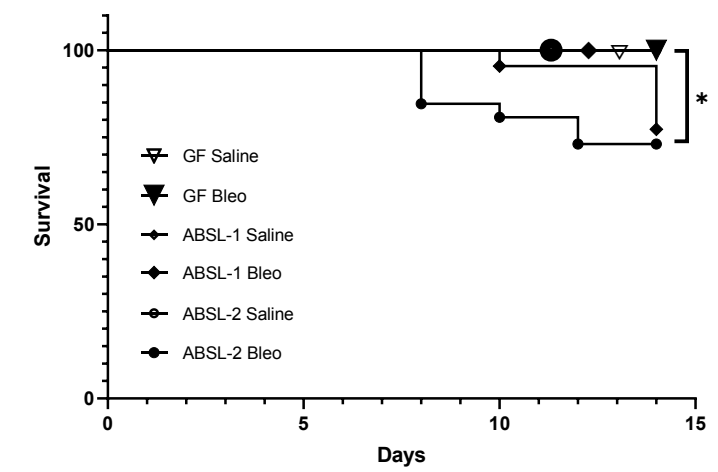

H\&E

Trichrome

ABSL-2

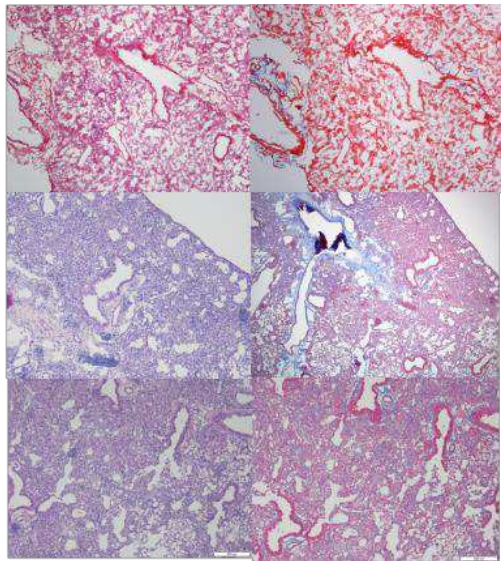

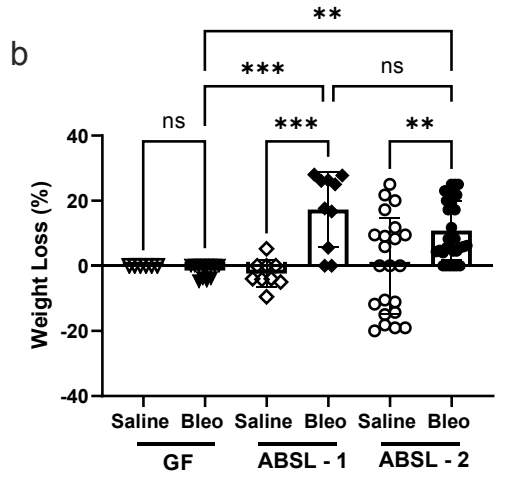

d

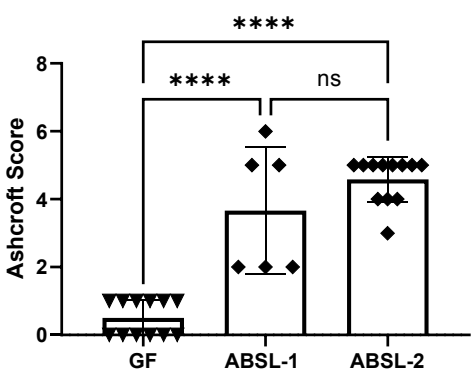

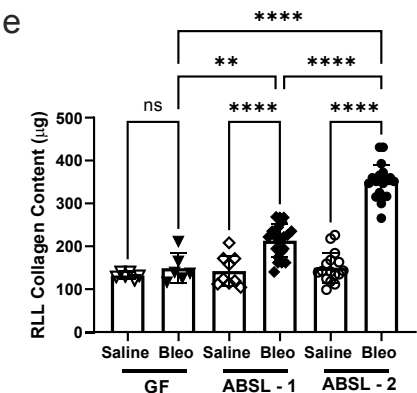

Figure 1. Germ-free mice are protected from lung fibrosis morbidity and mortality.

(a) Survival curve of mice housed in gnotobiotic, ABSL-1, or ABSL-2 facilities and intranasally inoculated with bleomycin.

(b) Body weight of mice in the housing and treatment cohorts was continuously measured and recorded daily from day 1 to day 14 .

c) Representative images for H\&E- and trichrome-stained lungs of GF, ABSL-1 and ABSL-2 mice, 14 days after bleomycin injury with Scale bar, $100 \mu \mathrm{m}$. (d) Ashcroft Scoring of trichrome-stained lungs by rearing environmentsfollowing bleomycin administration to mice $(n=6-12$ per cohort).

e) Soluble collagen content of RLL of mice exposed to bleomycin or saline treatment and housed in gnotobiotic, ABSL-1, or ABSL-2 facilities by Sircol assay; Data are reported as mean \pm SD with each dot representing an individual mouse, $n=6-25$ mice. Statistical significance was assessed using a one-way ANOVA with Tukey's multiple comparison's test. ${ }^{*} \mathrm{P}<0.05 * * \mathrm{P}<0.01$, $* * * \mathrm{P}<$ $0.001 * * * * \mathrm{P}<0.0001$; GF: Germ-free, ABSL-1: Animal Biosafety Level 1, ABSL-2: Animal Biosafety Level 2, ns = no significance; RLL: right lower lobe. 


\section{Figure 2}
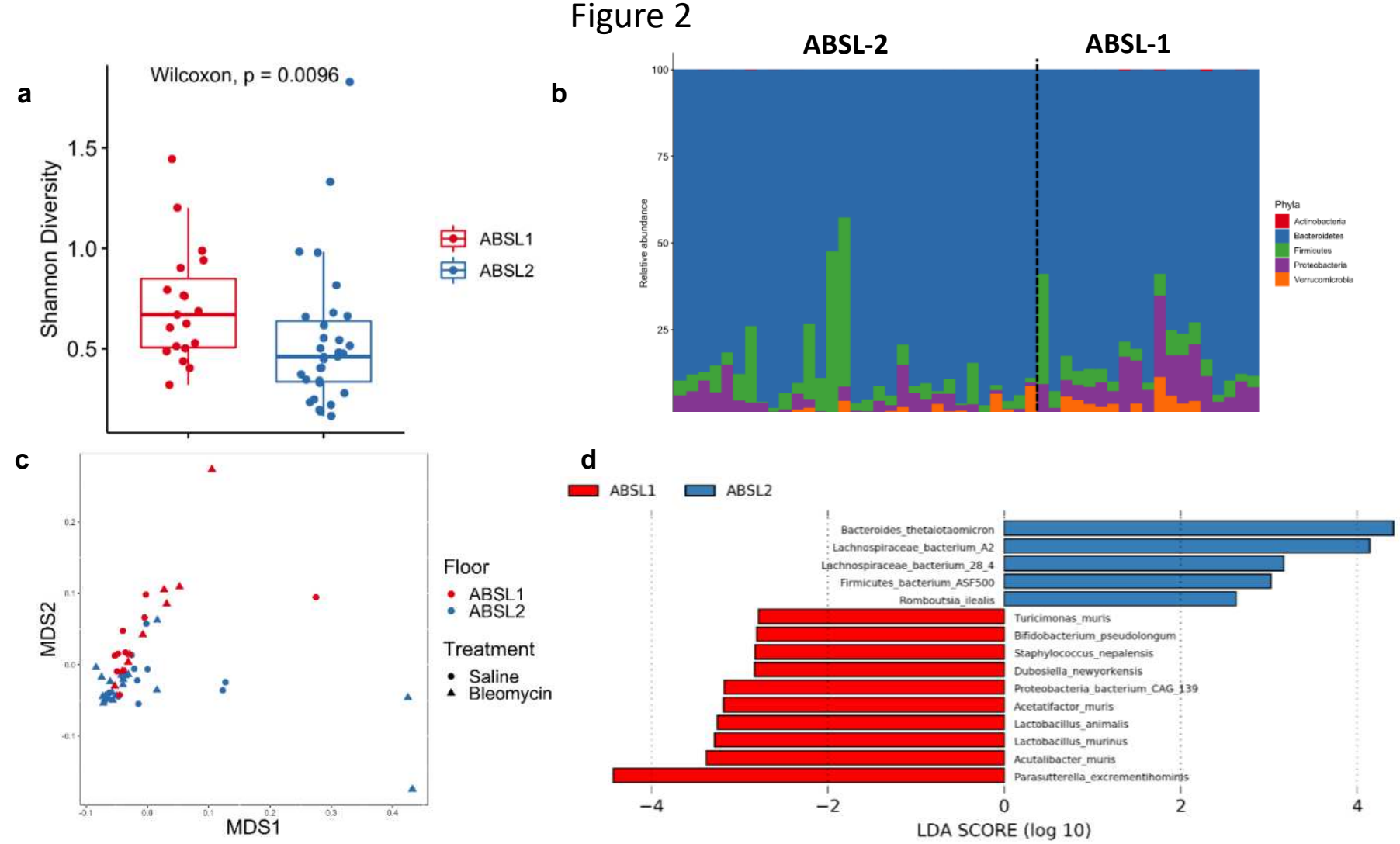

Figure 2. Increased gut microbial diversity is associated with better outcomes in murine model of fibrosis. (a) Shannon diversity index scores for ABSL-1 and ABSL-2 mice (b) Phyla-level composition of the gut microbiome of ABSL-1 and ABSL-2 mice (c) Nonlinear multidimensional scaling (MDS) plot showing differences in microbial taxonomic composition based on Bray-Curtis dissimilarities (d) Results of linear discriminant analysis showing taxa that are overrepresented in ABSL-1 or ABSL-2 mice 
Figure 3

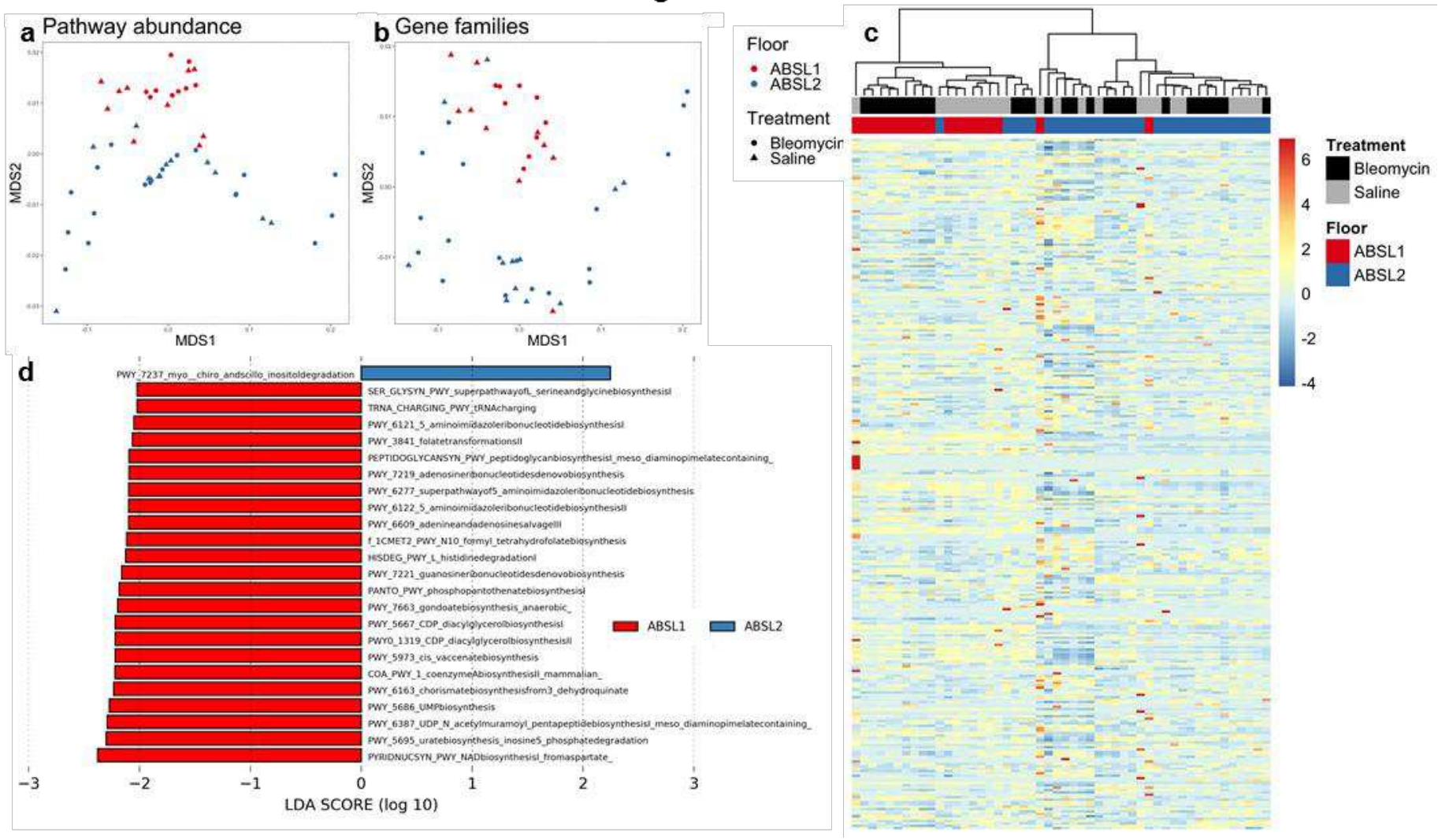

Figure 3. Functional gene profiles are significantly associated with rearing environment and treatment. (a) MetaCyc reaction pathway abundance and (b) gene family abundance profiles using nonlinear multidimensional scaling (MDS) plots based on Jaccard distance matrices (c) Heatmap showing normalized abundances of MetaCyc reaction pathway abundances between rearing environment and treatments (d) Results of linear discriminant analysis showing MetaCyc reaction pathways that are overrepresented in ABSL1 or ABSL2 mice 
Figure 4

$\mathrm{b}$
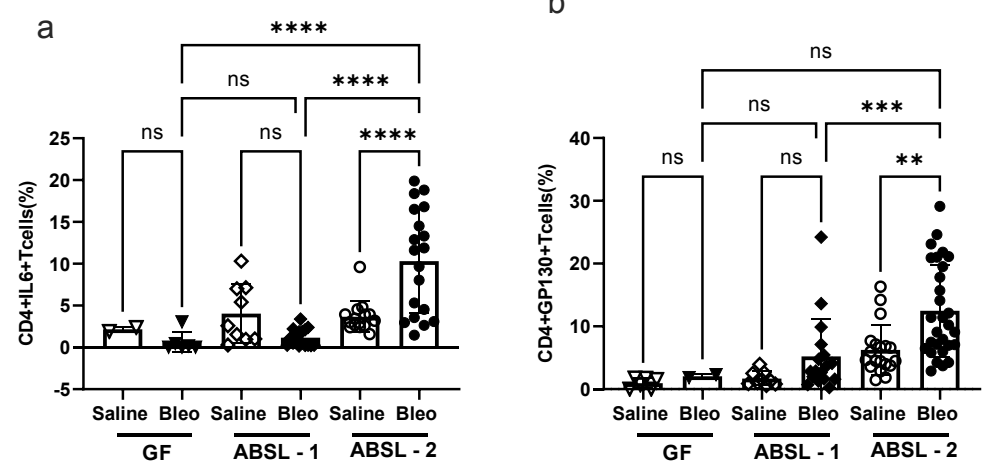

C

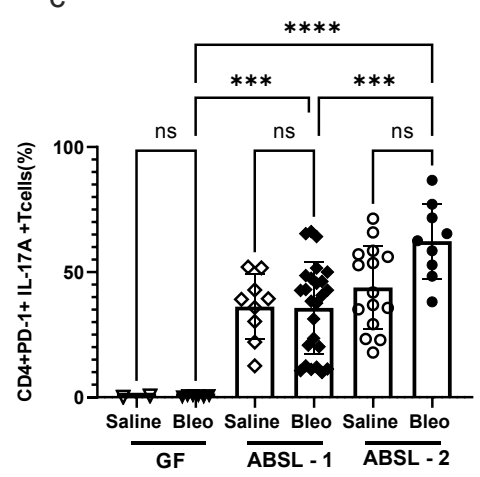

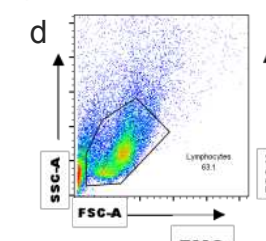
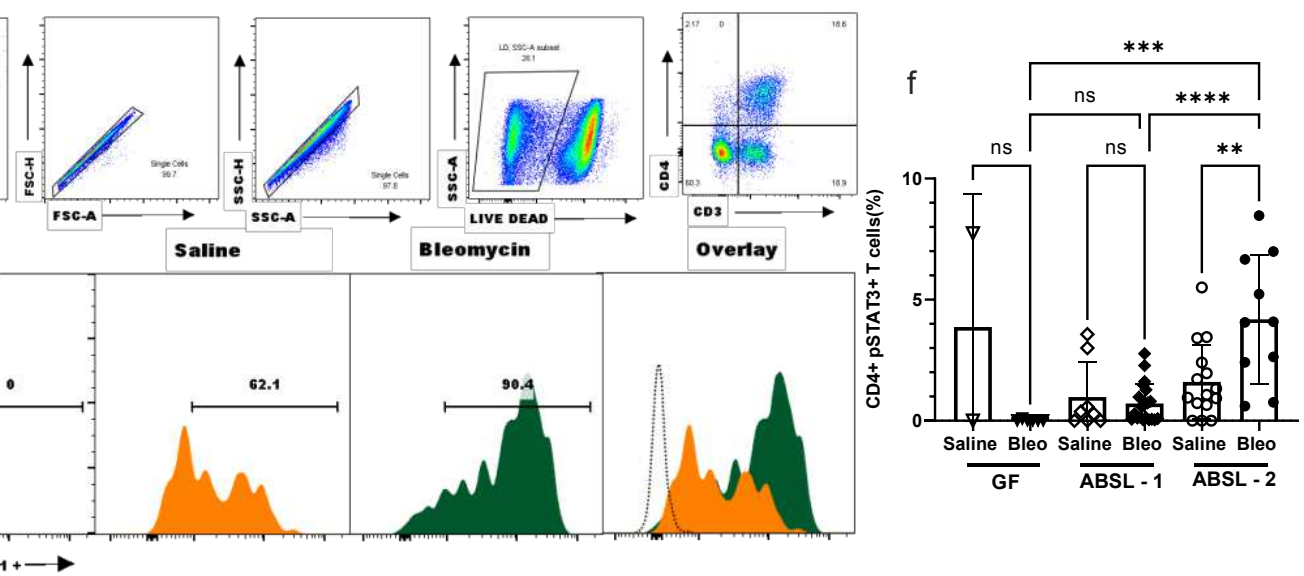

Figure 4. Germ-free mice do not mount a proinflammatory response upon bleomycin treatment.

Flow cytometric analysis of T cells from single cell lung suspensions at day 14. Single cell lung suspensions were obtained from mice housed in gnotobiotic, ABSL-1, or ABSL-2 facilities (GF, ABSL-1 and ABSL-2). Flow cytometry analysis was conducted for CD4+ (a) IL-6 (b) gp130 (e) PD-1+ IL-17A+ (d) gating strategy (e) representative histograms and (f) pSTAT3 ${ }^{\text {Y705 }}$ expression on T cells. Comparisons between cohorts were performed using one-way ANOVA with Tukey's post-hoc. $* \mathrm{P}<0.05, * * \mathrm{P}<0.01, * * * \mathrm{P}<0.001, * * * * \mathrm{P}<0.0001$, ns: no significance. Bars are mean $\pm \mathrm{SD}$; each symbol represents an individual mouse, $\mathrm{n}=2-19$ mice. 
Figure 5

a

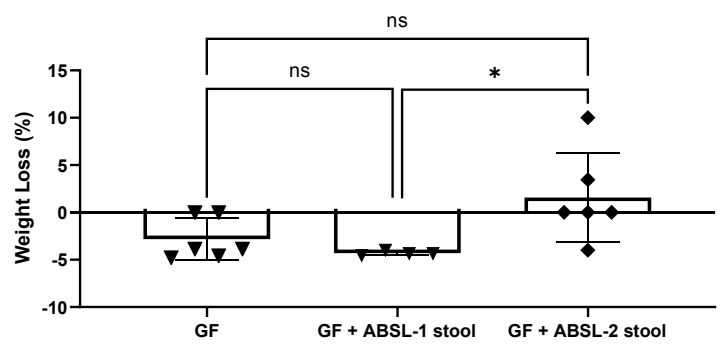

b

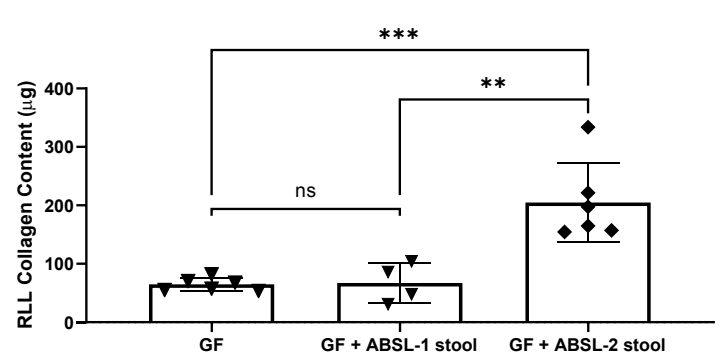

Figure 5. Reconstitution of an ABSL-2 microbiome in GF mice restores lung fibrosis morbidity. Germ-free mice were gavaged with ABSL-1 or ABSL-2 fecal slurries and given bleomycin to induce lung fibrosis (a) Weight loss at day 14 and (b) RLL collagen content by Sircol assay were determined across the gavage treatment groups. Each symbol represents an individual mouse. Statistical significance was assessed using a one-way ANOVA with Tukey's post-hoc. ${ }^{*} \mathrm{P}<0.05$, $* * \mathrm{P}<0.01, * * * \mathrm{P}<0.001, \mathrm{~ns}=$ no significance 
Figure 6

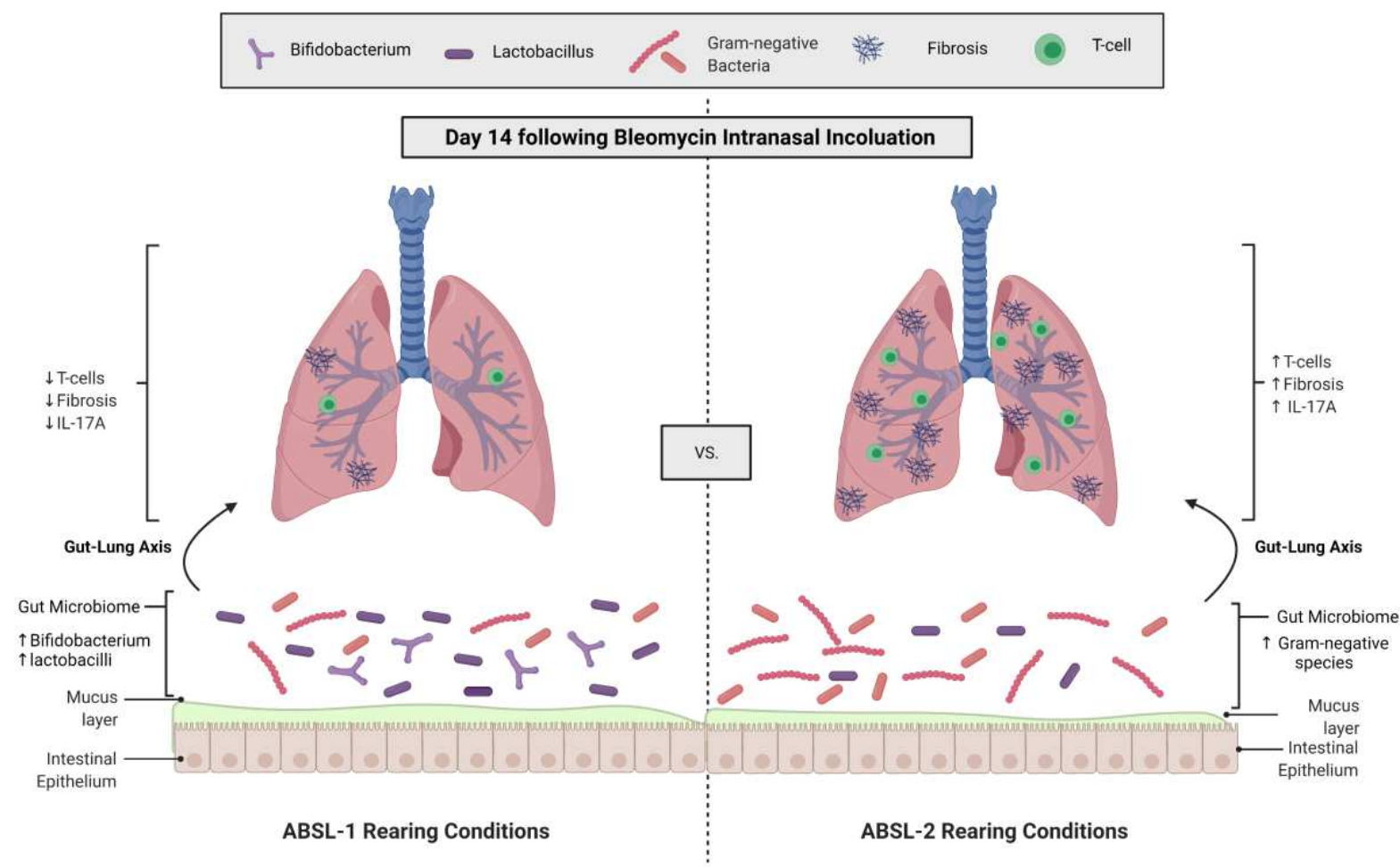

"Created with BioRender.com"

Figure 6. Model of gut contribution to fibrotic lung disease. 


\section{References}

1 Stokholm, J. et al. Maturation of the gut microbiome and risk of asthma in childhood. Nat Commun 9, 141, doi:10.1038/s41467-017-02573-2 (2018).

2 Travis, W. D. et al. An official American Thoracic Society/European Respiratory Society statement: Update of the international multidisciplinary classification of the idiopathic interstitial pneumonias. Am J Respir Crit Care Med 188, 733-748, doi:10.1164/rccm.201308-1483ST (2013).

3 Raghu, G. et al. Diagnosis of Idiopathic Pulmonary Fibrosis. An Official ATS/ERS/JRS/ALAT Clinical Practice Guideline. Am J Respir Crit Care Med 198, e44-e68, doi:10.1164/rccm.201807-1255ST (2018).

4 Strongman, H., Kausar, I. \& Maher, T. M. Incidence, Prevalence, and Survival of Patients with Idiopathic Pulmonary Fibrosis in the UK. Adv Ther 35, 724-736, doi:10.1007/s12325-018-0693-1 (2018).

$5 \quad$ Swigris, J. J. et al. Sarcoidosis-related mortality in the United States from 1988 to 2007. Am J Respir Crit Care Med 183, 1524-1530, doi:10.1164/rccm.201010-16790C (2011).

6 Huang, Y. et al. Microbes Are Associated with Host Innate Immune Response in Idiopathic Pulmonary Fibrosis. Am J Respir Crit Care Med 196, 208-219, doi:10.1164/rccm.201607-15250C (2017).

7 O'Dwyer, D. N. et al. Lung Microbiota Contribute to Pulmonary Inflammation and Disease Progression in Pulmonary Fibrosis. Am J Respir Crit Care Med 199, 1127-1138, doi:10.1164/rccm.201809-16500C (2019).

8 Andréasson, K., Alrawi, Z., Persson, A., Jönsson, G. \& Marsal, J. Intestinal dysbiosis is common in systemic sclerosis and associated with gastrointestinal and extraintestinal features of disease. Arthritis Res Ther 18, 278, doi:10.1186/s13075-016-1182-z (2016).

9 Zhou, Y., Chen, L., Sun, G., Li, Y. \& Huang, R. Alterations in the gut microbiota of patients with silica-induced pulmonary fibrosis. J Occup Med Toxicol 14, 5, doi:10.1186/s12995-019-0225-1 (2019).

10 Budden, K. F. et al. Emerging pathogenic links between microbiota and the gut-lung axis. Nat Rev Microbiol 15, 55-63, doi:10.1038/nrmicro.2016.142 (2017).

11 Chioma, O. S., Hesse, L. E., Chapman, A. \& Drake, W. P. Role of the Microbiome in Interstitial Lung Diseases. Front Med (Lausanne) 8, 595522, doi:10.3389/fmed.2021.595522 (2021).

12 Liévin-Le Moal, V. \& Servin, A. L. The front line of enteric host defense against unwelcome intrusion of harmful microorganisms: mucins, antimicrobial peptides, and microbiota. Clin Microbiol Rev 19, 315-337, doi:10.1128/cmr.19.2.315-337.2006 (2006).

13 Ruiz, P. A., Hoffmann, M., Szcesny, S., Blaut, M. \& Haller, D. Innate mechanisms for Bifidobacterium lactis to activate transient pro-inflammatory host responses in intestinal epithelial cells after the colonization of germ-free rats. Immunology 115, 441-450, doi:10.1111/j.1365-2567.2005.02176.x (2005).

14 Schirmer, M. et al. Linking the Human Gut Microbiome to Inflammatory Cytokine Production Capacity. Cell 167, 1125-1136.e1128, doi:10.1016/j.cell.2016.10.020 (2016).

15 Epstein Shochet, G., Brook, E., Bardenstein-Wald, B. \& Shitrit, D. TGF- $\beta$ pathway activation by idiopathic pulmonary fibrosis (IPF) fibroblast derived soluble factors is mediated by IL-6 transsignaling. Respir Res 21, 56, doi:10.1186/s12931-020-1319-0 (2020).

16 Le, T. T. et al. Blockade of IL-6 Trans signaling attenuates pulmonary fibrosis. J Immunol 193, 37553768, doi:10.4049/jimmunol.1302470 (2014). 
17 O'Donoghue, R. J. et al. Genetic partitioning of interleukin-6 signalling in mice dissociates Stat3 from Smad3-mediated lung fibrosis. EMBO Mol Med 4, 939-951, doi:10.1002/emmm.201100604 (2012).

18 Chen, L. et al. Microbiota Metabolite Butyrate Differentially Regulates Th1 and Th17 Cells' Differentiation and Function in Induction of Colitis. Inflamm Bowel Dis 25, 1450-1461, doi:10.1093/ibd/izz046 (2019).

19 Furusawa, Y. et al. Commensal microbe-derived butyrate induces the differentiation of colonic regulatory T cells. Nature 504, 446-450, doi:10.1038/nature12721 (2013).

20 Vester-Andersen, M. K. et al. Increased abundance of proteobacteria in aggressive Crohn's disease seven years after diagnosis. Sci Rep 9, 13473, doi:10.1038/s41598-019-49833-3 (2019).

21 Sverrild, A. et al. Eosinophilic airway inflammation in asthmatic patients is associated with an altered airway microbiome. J Allergy Clin Immunol 140, 407-417.e411, doi:10.1016/j.jaci.2016.10.046 (2017).

22 Bernasconi, E. et al. Airway Microbiota Determines Innate Cell Inflammatory or Tissue Remodeling Profiles in Lung Transplantation. Am J Respir Crit Care Med 194, 1252-1263, doi:10.1164/rccm.201512-24240C (2016).

23 Arpaia, N. et al. Metabolites produced by commensal bacteria promote peripheral regulatory Tcell generation. Nature 504, 451-455, doi:10.1038/nature12726 (2013).

$24 \mathrm{He}$, B. et al. Lactobacillus reuteri Reduces the Severity of Experimental Autoimmune Encephalomyelitis in Mice by Modulating Gut Microbiota. Front Immunol 10, 385, doi:10.3389/fimmu.2019.00385 (2019).

25 Rizzo, A., Losacco, A., Carratelli, C. R., Domenico, M. D. \& Bevilacqua, N. Lactobacillus plantarum reduces Streptococcus pyogenes virulence by modulating the IL-17, IL-23 and Toll-like receptor 2/4 expressions in human epithelial cells. Int Immunopharmacol 17, 453-461, doi:10.1016/j.intimp.2013.07.005 (2013).

26 Michael, D. R. et al. A randomised controlled study shows supplementation of overweight and obese adults with lactobacilli and bifidobacteria reduces bodyweight and improves well-being. Sci Rep 10, 4183, doi:10.1038/s41598-020-60991-7 (2020).

27 Elijovich, F. et al. The Gut Microbiome, Inflammation, and Salt-Sensitive Hypertension. Curr Hypertens Rep 22, 79, doi:10.1007/s11906-020-01091-9 (2020).

28 Brandsma, E. et al. A Proinflammatory Gut Microbiota Increases Systemic Inflammation and Accelerates Atherosclerosis. Circ Res 124, 94-100, doi:10.1161/circresaha.118.313234 (2019).

29 Ivanov, II et al. Specific microbiota direct the differentiation of IL-17-producing T-helper cells in the mucosa of the small intestine. Cell Host Microbe 4, 337-349, doi:10.1016/j.chom.2008.09.009 (2008).

30 Zeng, Q. et al. Discrepant gut microbiota markers for the classification of obesity-related metabolic abnormalities. Sci Rep 9, 13424, doi:10.1038/s41598-019-49462-w (2019).

31 Cozier, Y. C. et al. Obesity and weight gain in relation to incidence of sarcoidosis in US black women: data from the Black Women's Health Study. Chest 147, 1086-1093, doi:10.1378/chest.141099 (2015).

32 Allan, E. et al. More diverse plant communities have higher functioning over time due to turnover in complementary dominant species. Proc Natl Acad Sci U S A 108, 17034-17039, doi:10.1073/pnas.1104015108 (2011).

33 McNaughton, S. J. Ecology of a Grazing Ecosystem: The Serengeti. Ecological Monographs 55, 259294, doi:https://doi.org/10.2307/1942578 (1985).

34 Tilman, D., Reich, P. B. \& Knops, J. M. Biodiversity and ecosystem stability in a decade-long grassland experiment. Nature 441, 629-632, doi:10.1038/nature04742 (2006). 
Wittebolle, L. et al. Initial community evenness favours functionality under selective stress. Nature 458, 623-626, doi:10.1038/nature07840 (2009).

36 Lozupone, C. A., Stombaugh, J. I., Gordon, J. I., Jansson, J. K. \& Knight, R. Diversity, stability and resilience of the human gut microbiota. Nature 489, 220-230, doi:10.1038/nature11550 (2012).

37 Taur, Y. et al. Reconstitution of the gut microbiota of antibiotic-treated patients by autologous fecal microbiota transplant. Sci Transl Med 10, doi:10.1126/scitranslmed.aap9489 (2018).

38 Khanna, S. et al. A Novel Microbiome Therapeutic Increases Gut Microbial Diversity and Prevents Recurrent Clostridium difficile Infection. J Infect Dis 214, 173-181, doi:10.1093/infdis/jiv766 (2016).

Alghetaa, H. et al. Resveratrol-mediated attenuation of superantigen-driven acute respiratory distress syndrome is mediated by microbiota in the lungs and gut. Pharmacol Res 167, 105548, doi:10.1016/j.phrs.2021.105548 (2021).

40 Liu, Y. et al. Fecal transplantation alleviates acute liver injury in mice through regulating Treg/Th17 cytokines balance. Sci Rep 11, 1611, doi:10.1038/s41598-021-81263-y (2021).

41 Bojanova, D. P. \& Bordenstein, S. R. Fecal Transplants: What Is Being Transferred? PLoS Biol 14, e1002503, doi:10.1371/journal.pbio.1002503 (2016).

42 Molyneaux, P. L. et al. The role of bacteria in the pathogenesis and progression of idiopathic pulmonary fibrosis. Am J Respir Crit Care Med 190, 906-913, doi:10.1164/rccm.201403-05410C (2014).

43 Lawson, W. E. et al. Endoplasmic reticulum stress enhances fibrotic remodeling in the lungs. Proc Natl Acad Sci U S A 108, 10562-10567, doi:10.1073/pnas.1107559108 (2011).

44 Celada, L. J. et al. PD-1 up-regulation on CD4(+) T cells promotes pulmonary fibrosis through STAT3-mediated IL-17A and TGF- $\beta 1$ production. Sci Transl Med 10, doi:10.1126/scitranslmed.aar8356 (2018).

45 Segata, N. et al. Metagenomic microbial community profiling using unique clade-specific marker genes. Nat Methods 9, 811-814, doi:10.1038/nmeth.2066 (2012).

46 Franzosa, E. A. et al. Species-level functional profiling of metagenomes and metatranscriptomes. Nat Methods 15, 962-968, doi:10.1038/s41592-018-0176-y (2018).

47 Jari Oksanen, F. G. B., Michael Friendly, Roeland Kindt, Pierre Legendre, Dan McGlinn, Peter R. Minchin, R. B. O'Hara, Gavin L. Simpson, Peter Solymos, M. Henry H. Stevens, Eduard Szoecs, Helene Wagner. Vol. R (2020).

48 Team, R. C. (https://www.R-project.org/, 2018).

49 Segata, N. et al. Metagenomic biomarker discovery and explanation. Genome Biol 12, R60, doi:10.1186/gb-2011-12-6-r60 (2011).

50 Brooks, M. E. K., Kasper; van Benthem, Koen J.; Magnusson, Arni; Berg, Casper W.; Nielsen, \& Anders; Skaug, H. J. M., Martin; Bolker, Benjamin M. glmmTMB balances speed and flexibility among packages for zero-inflated generalized linear mixed modeling. The R journal 9, 378 - 400, doi:https://doi.org/10.3929/ethz-b-000240890 (2017).

51 Strimmer, B. K. a. K. Vol. R (CRAN, 2020).

52 Herazo-Maya, J. D. et al. Validation of a 52-gene risk profile for outcome prediction in patients with idiopathic pulmonary fibrosis: an international, multicentre, cohort study. Lancet Respir Med 5, 857-868, doi:10.1016/s2213-2600(17)30349-1 (2017).

53 Collins, S. L. et al. Vaccinia vaccine-based immunotherapy arrests and reverses established pulmonary fibrosis. JCl Insight 1, e83116, doi:10.1172/jci.insight.83116 (2016). 
injury

Supplementary Figure 1

a

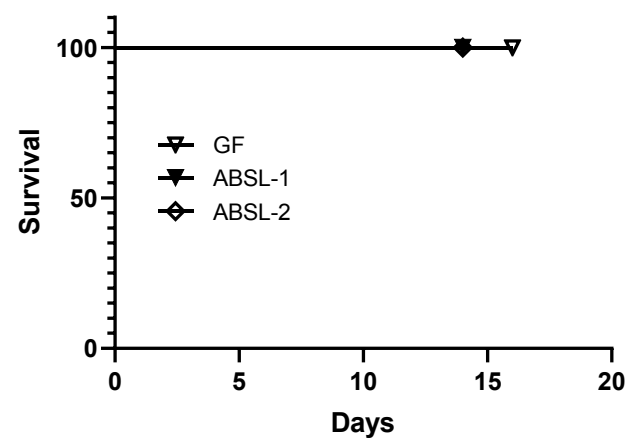

C

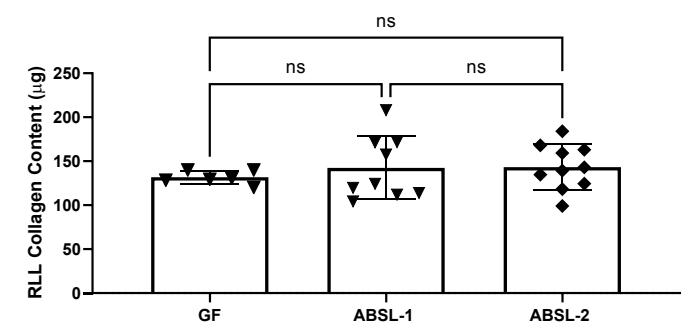

b

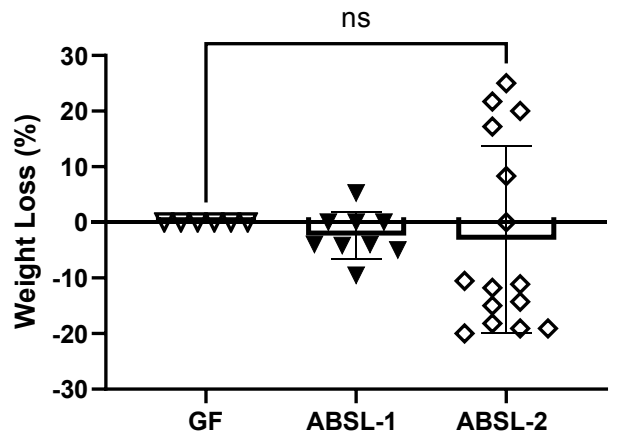

Supplemental Figure 1. Housing conditions does not impact morbidity and mortality during saline treatment. (a) Survival curve of mice housed in 3 separate facilities and intranasally inoculated with saline. (b) Weight loss of mice in the saline treatment cohorts (c) Sircol assay of mice housed in 3 separate facilities and treated with saline (control group); Data are reported as mean $\pm \mathrm{SD}$ with each dot representing an individual mouse. Statistical significance was assessed using a one-way ANOVA with Sidak's multiple comparison's test. $* \mathrm{P}<0.05 * * \mathrm{P}<0.01,{ }^{* * * *} \mathrm{P}<0.0001 ; \mathrm{ns}=$ no significance; RLL: right lower lobe. Data in (b) and (c) are shown as part of Figure 1. 
Supplementary Figure 2

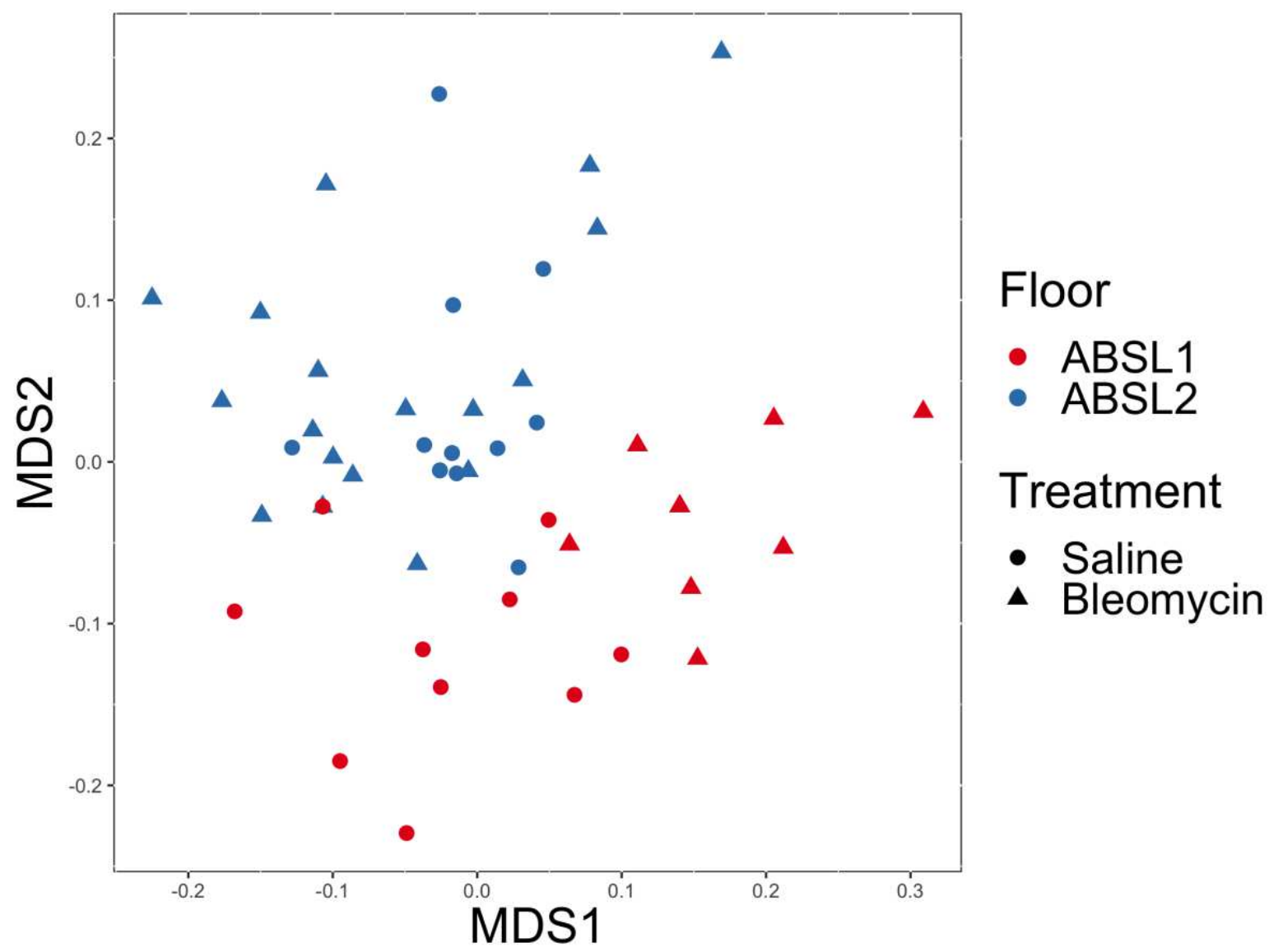

Supplemental Figure 2. Rearing environment alters gut microbiota composition. Nonlinear multidimensional scaling (MDS) plot showing differences in microbial taxonomic composition based on Jaccard index 

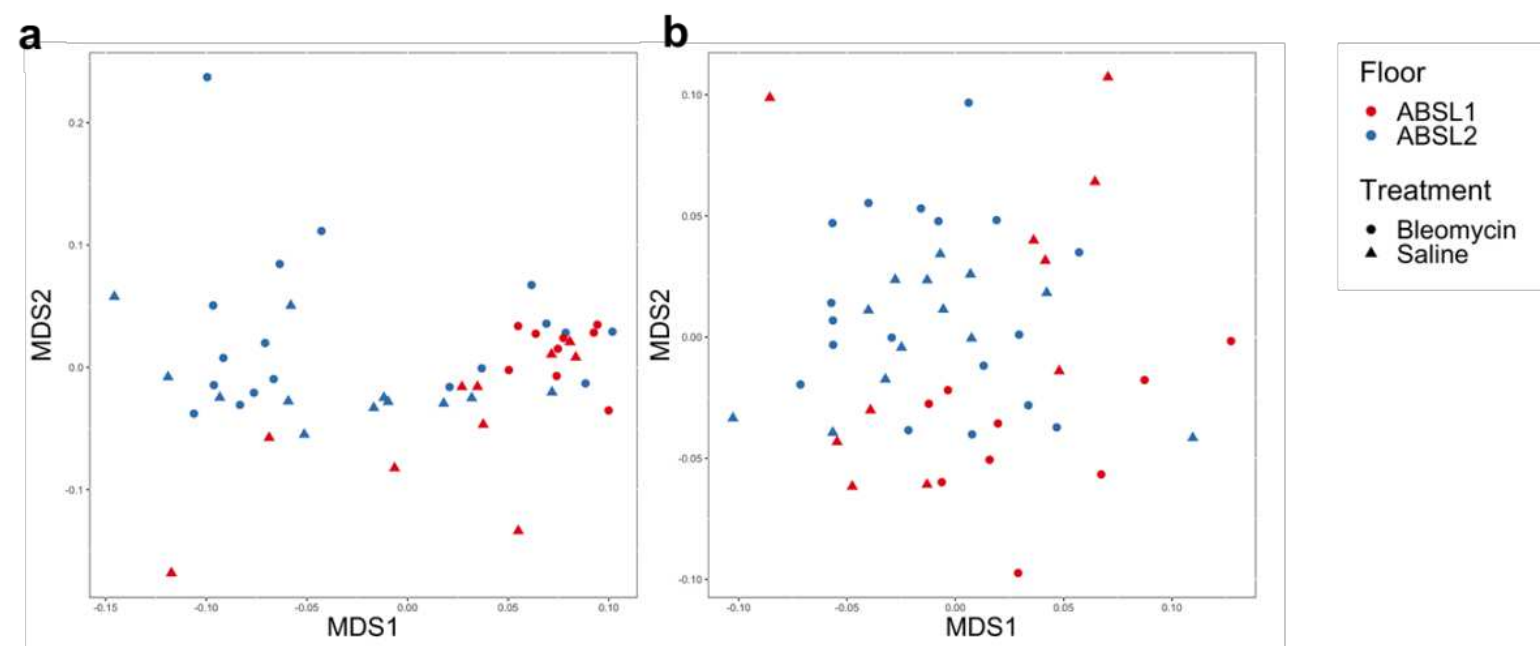

Supplemental Figure 3. Functional profiles are not different between floor or treatment when using Bray-Curtis dissimilarity matrices (a) Gene family abundance and (b) MetaCyc reaction pathway abundance profiles based on Bray-Curtis dissimilarity matrices. 
a

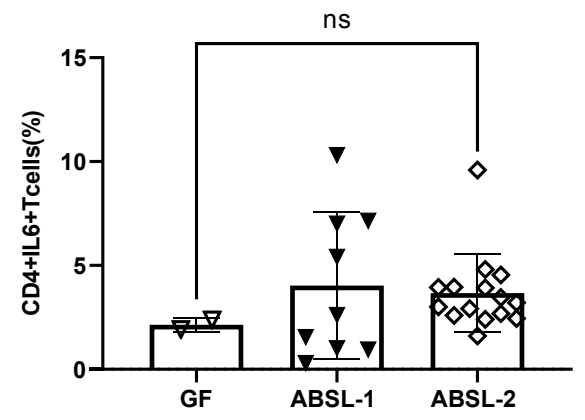

b

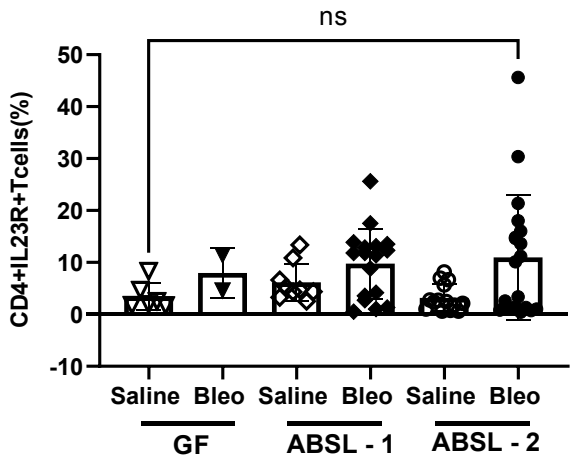

Supplemental Figure 4. Gut microbial diversity and taxa associate with inflammatory pulmonary lung responses. Single cell lung suspensions were obtained from mice housed in three different facilities (GF, ABSL-1 and ABSL-2). Flow cytometry analysis was conducted for (a) IL-6 and (b) IL-23R expression on CD4+T cells. Statistical significance was assessed using a one-way ANOVA with Tukey's multiple comparison's test. $* \mathrm{P}<0.05, * * \mathrm{P}<0.01$, ****P $<$ $0.0001, \mathrm{~ns}=$ no significance $\mathrm{n}=2-19$. 
Supplementary Figure 5a

a Germ Free $\left(\mathrm{CD}^{+}{ }^{+} \mathrm{IL}-6^{+}{ }^{+}\right.$T Cells $\left.(\%)\right)$

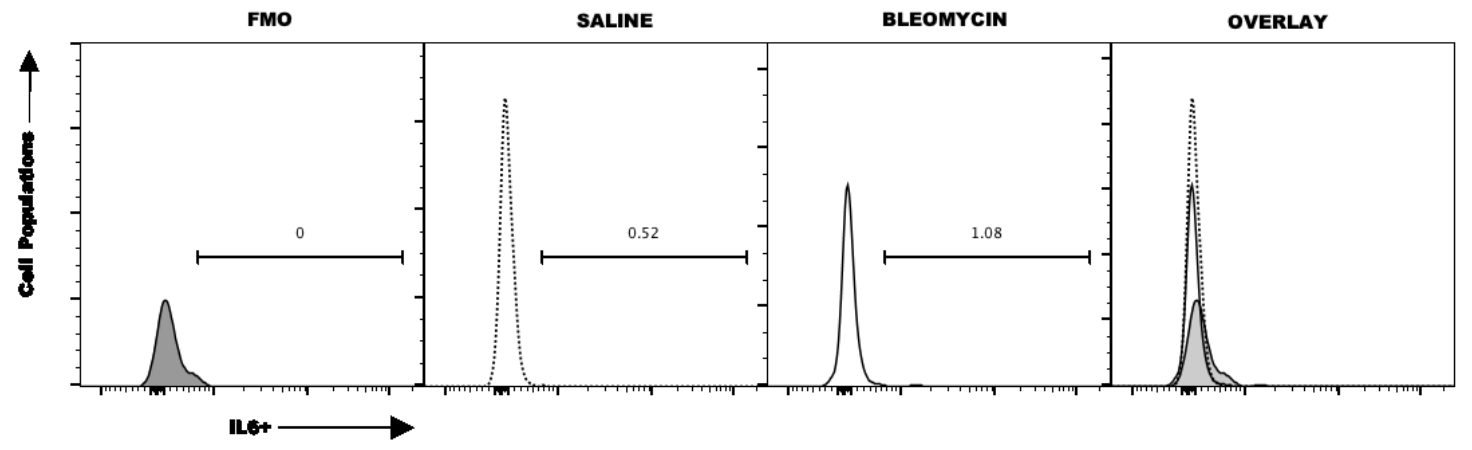

b ABSL-1 (CD4 ${ }^{+}$IL- $6{ }^{+}$T Cells (\%))

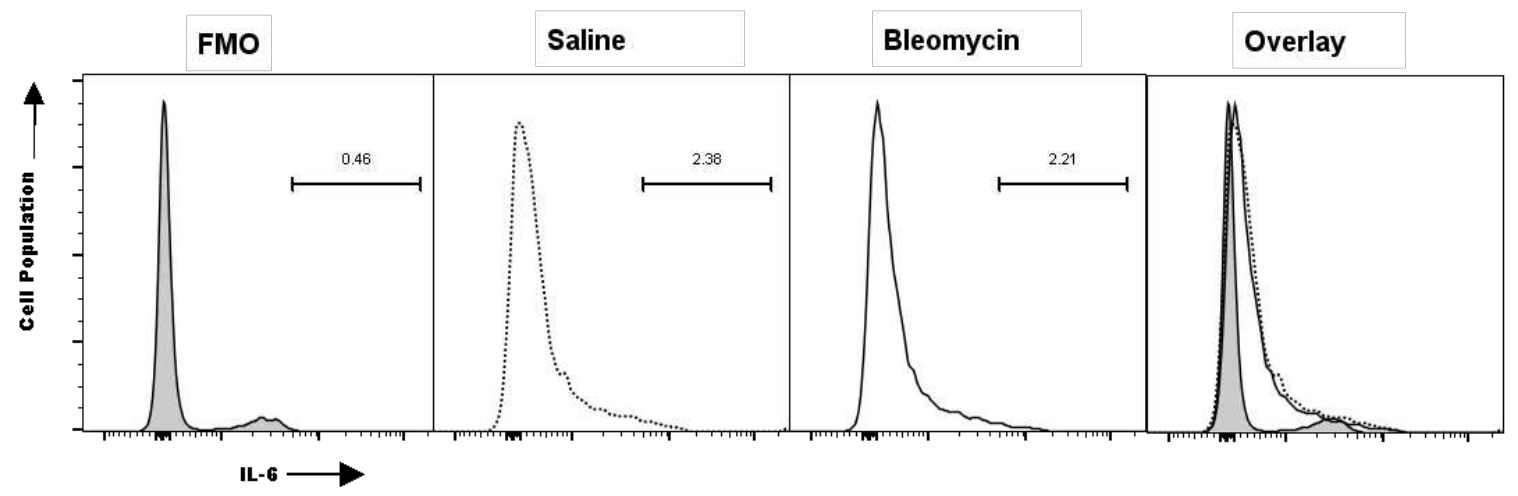

c ABSL-2 $\left(\mathrm{CD}^{+}{ }^{+} \mathrm{IL}-6^{+}\right.$T Cells (\%))

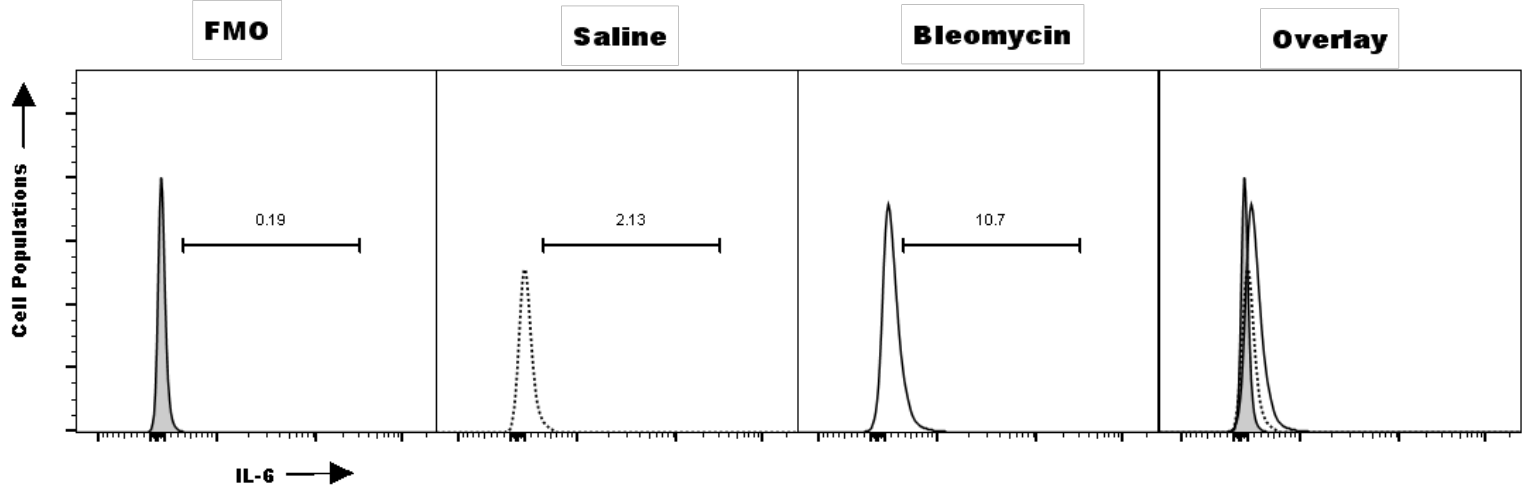

Supplementary Fig. 5a: Representative histogram depicting IL-6 expression on CD4+ T cells in GF, ABSL-1 and ABSL-2 saline and bleomycin treated mice

a) Representative histogram depicting IL-6 expression on CD4+ T cells in GF mice b) Representative histogram depicting IL- 6 expression on CD4+ T cells in ABSL-1 mice c) Representative histogram depicting IL-6 expression on CD4+ T cells in ABSL-2 mice. GF: Germ-free, ABSL-1: Animal Biosafety Level 1, ABSL-2: Animal Biosafety Level 2 
Supplementary Figure 5b

a Germ Free $\left(\mathrm{CD}^{+} \mathrm{GP}-130^{+} \mathrm{T}\right.$ Cells $\left.(\%)\right)$

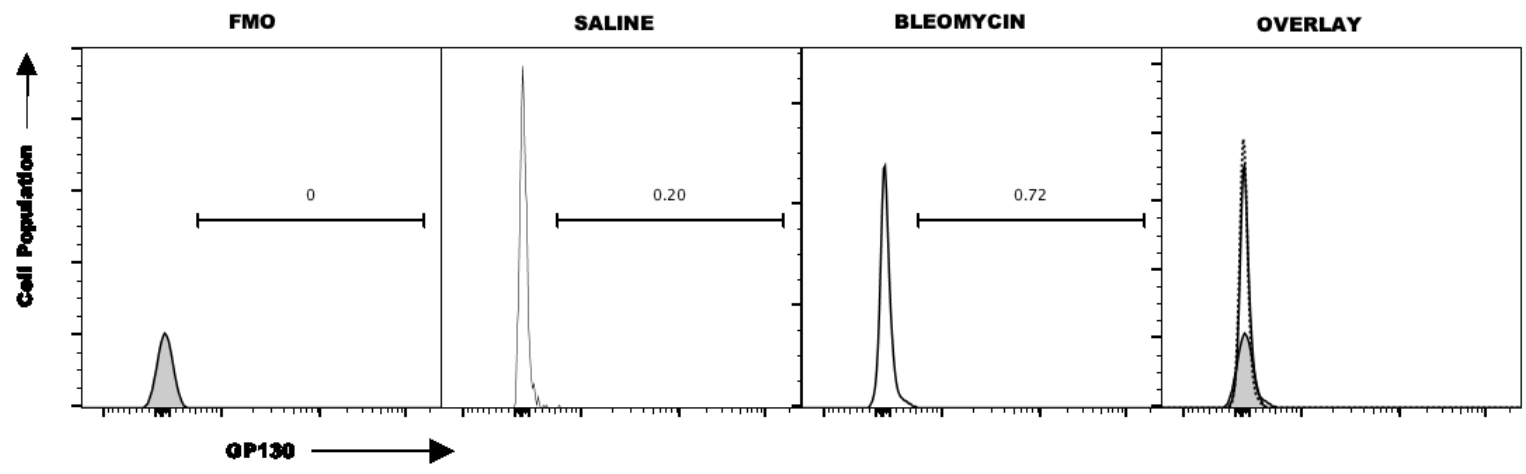

b ABSL-1 (CD4 ${ }^{+} \mathrm{GP}-130^{+} \mathrm{T}$ Cells (\%))

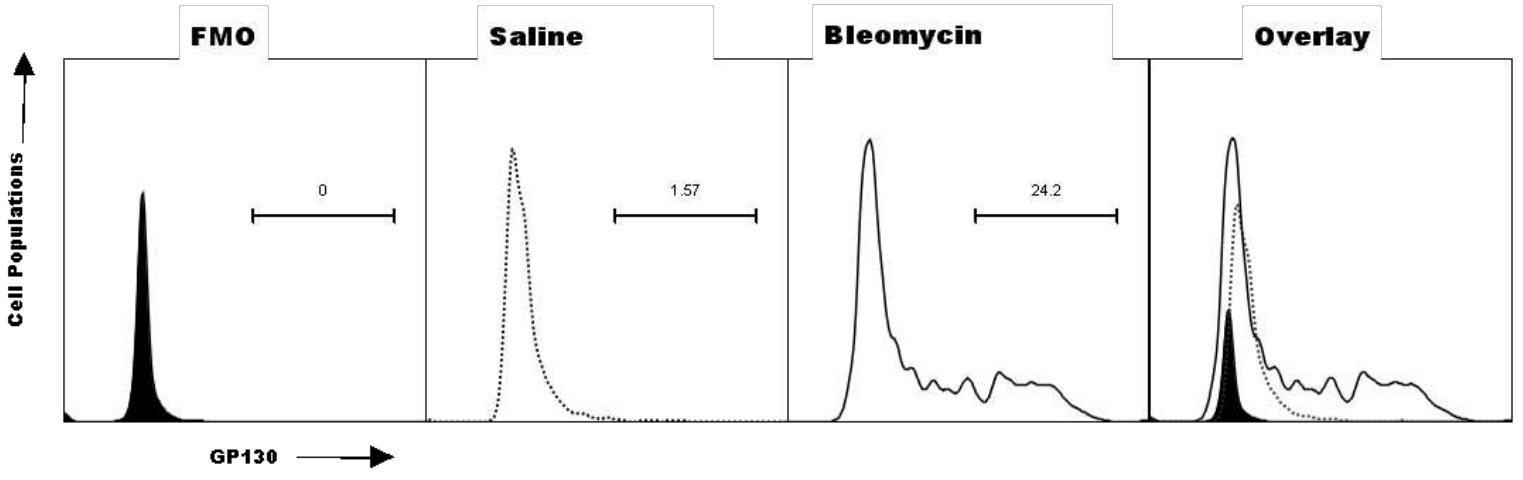

c ABSL-2 (CD4 ${ }^{+} \mathrm{GP}-130^{+} \mathrm{T}$ Cells $\left.(\%)\right)$

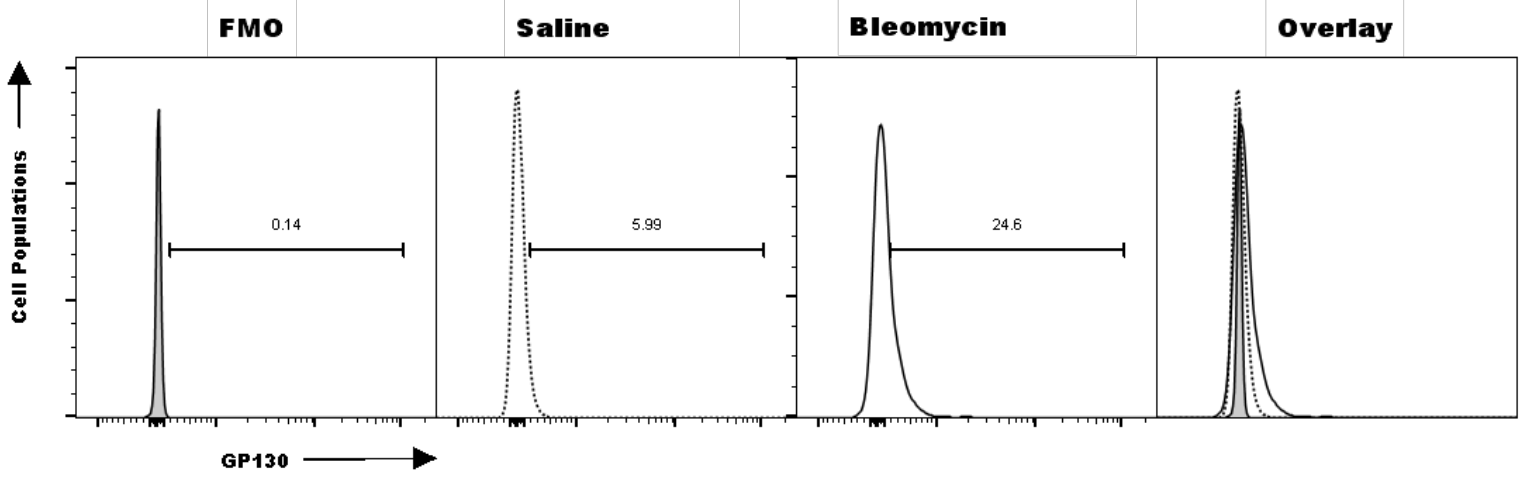

Supplementary Fig. 5b: Representative histogram depicting GP130 expression on CD4+ T cells in GF, ABSL-1 and ABSL-2 saline and bleomycin treated mice

a) Representative histogram depicting GP130 expression on CD4+ T cells in GF mice b) Representative histogram depicting GP130 expression on CD4+ T cells in ABSL-1 mice c) Representative histogram depicting GP130 expression on CD4+ T cells in ABSL-2 mice GF: Germ-free, ABSL-1: Animal Biosafety Level 1, ABSL-2: Animal Biosafety Level 2 
Germ Free (CD4 ${ }^{+}$PD-1+ IL-17A ${ }^{+}$T Cells (\%))

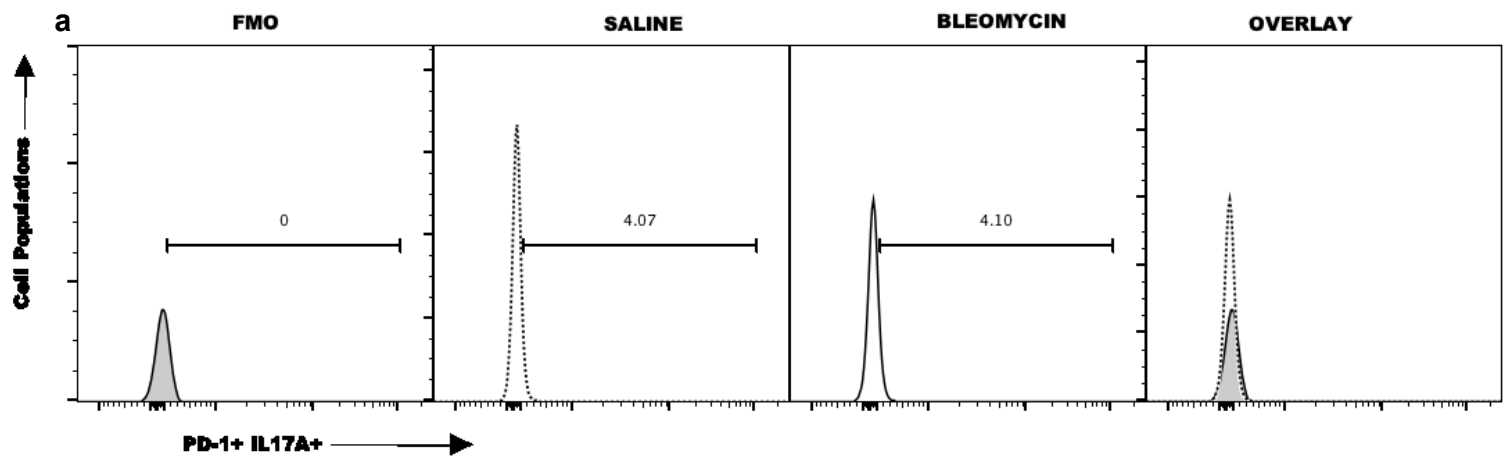

b ABSL-1 (CD4 ${ }^{+}$PD-1+ IL-17A ${ }^{+}$T Cells (\%))

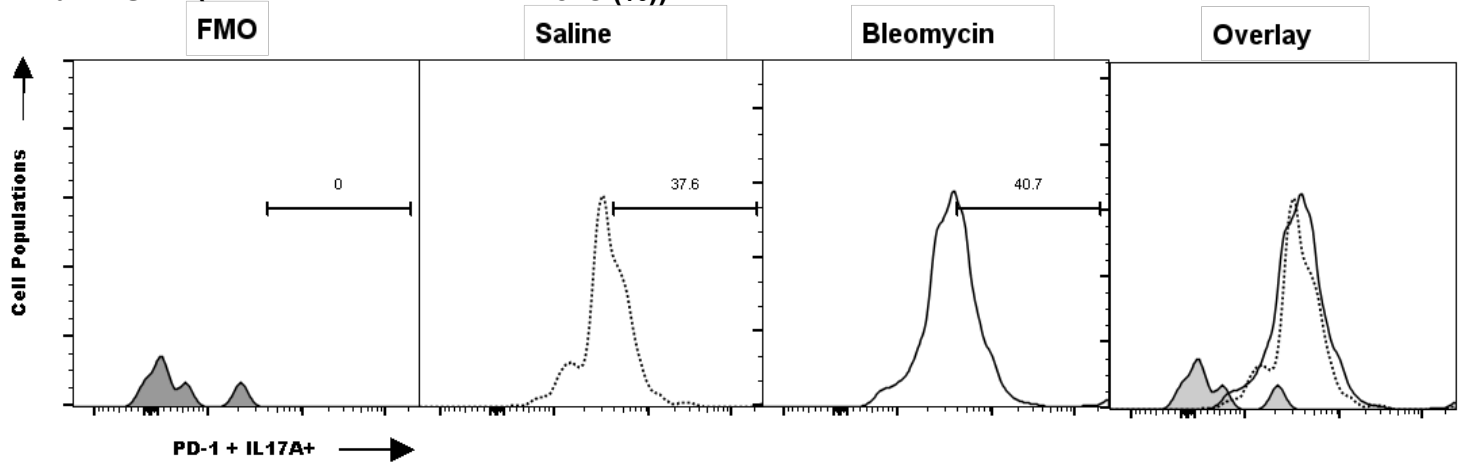

C ABSL-2 (CD4 ${ }^{+}$PD-1+ IL-17A ${ }^{+}$T Cells (\%))

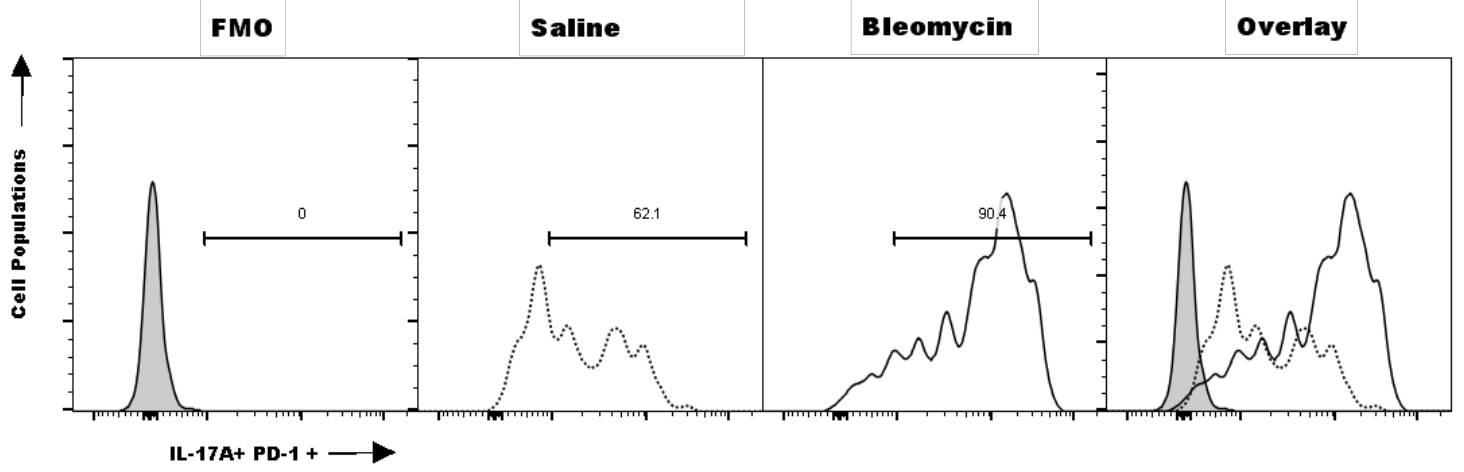

Supplementary Fig. 5c: Representative histogram depicting PD-1+ IL-17A+ expression on CD4+ $T$ cells in GF, ABSL-1 and ABSL-2 saline and bleomycin treated mice. a)

Representative histogram depicting PD-1+ IL-17A+ expression on CD4+ T cells in GF mice b) Representative histogram depicting PD-1+ IL-17A+ expression on CD4+ T cells in ABSL-1 mice c) Representative histogram depicting PD-1+ IL-17A+ expression on CD4+ $\mathrm{T}$ cells in ABSL-2 mice. GF: Germ-free, ABSL-1: Animal Biosafety Level 1, ABSL-2: Animal Biosafety Level 2 
Supplemental Table 1. Antibodies used in this study

\begin{tabular}{|l|l|l|l|l|}
\hline Antibody & $\begin{array}{l}\text { Vendor Catalog } \\
\text { No }\end{array}$ & $\begin{array}{l}\text { Host organism; } \\
\text { antibody type }\end{array}$ & Clone & $\begin{array}{l}\text { Conc } \\
(\boldsymbol{\mu g}) / \mathbf{v o l u m e} \\
(\boldsymbol{\mu L}) \mathbf{p e r} \text { test* }\end{array}$ \\
\hline $\begin{array}{l}\text { CD3 Alexa } \\
\text { Fluor 700 }\end{array}$ & $\begin{array}{l}\text { Biolegend } \\
100216\end{array}$ & Rat Monoclonal & $17 \mathrm{~A} 2$ & $0.25 \mu \mathrm{g} / \mu \mathrm{L}$ \\
\hline CD4 APC-Cy7 & $\begin{array}{l}\text { Biolegend } \\
100414\end{array}$ & $\begin{array}{l}\text { Mouse } \\
\text { Monoclonal }\end{array}$ & GK1.5 & $0.1 \mu \mathrm{g} / \mu \mathrm{L}$ \\
\hline IL-6 APC & $\begin{array}{l}\text { Biolegend } \\
504808\end{array}$ & Rat Monoclonal & MP5-20F3 & $0.04 \mu \mathrm{g} / \mu \mathrm{L}$ \\
\hline GP-130 PE & $\begin{array}{l}\text { Biolegend } \\
149404\end{array}$ & Rat Monoclonal & $4 \mathrm{H} 1 \mathrm{~B} 35$ & $0.02 \mu \mathrm{g} / \mu \mathrm{L}$ \\
\hline PD-1 PE-Cy7 & $\begin{array}{l}\text { Biolegend } \\
135216\end{array}$ & Rat Monoclonal & $29 \mathrm{~F} .1 \mathrm{~A} 12$ & $0.1 \mu \mathrm{g} / \mu \mathrm{L}$ \\
\hline IL-17A PE & $\begin{array}{l}\text { Biolegend } \\
506904\end{array}$ & Rat Monoclonal & TCII-18H10.1 & $0.1 \mu \mathrm{g} / \mu \mathrm{L}$ \\
\hline $\begin{array}{l}\text { STAT3 (pY703) } \\
\text { Pacific Blue }\end{array}$ & BD 560312 & $\begin{array}{l}\text { Mouse } \\
\text { Monoclonal }\end{array}$ & $4 / \mathrm{P}-\mathrm{STAT3}$ & $10 \mu \mathrm{g} / \mu \mathrm{L}$ \\
\hline IL-23R APC & $\begin{array}{l}\text { Biolegend } \\
150906\end{array}$ & Rat Monoclonal & $12 \mathrm{~B} 2 \mathrm{~B} 64$ & $0.02 \mu \mathrm{g} / \mu \mathrm{L}$ \\
\hline
\end{tabular}

${ }^{*}$ Test $=1$ million cells in $50 \mu 1$ of staining buffer 
